\title{
Metalloproteinase PAPP-A regulation of IGF-1 contributes to polycystic kidney disease pathogenesis
}

\author{
Sonu Kashyap, ${ }^{1}$ Kyaw Zaw Hein, ${ }^{1}$ Claudia C.S. Chini, ${ }^{1}$ Jorgo Lika, ${ }^{1}$ Gina M. Warner, ${ }^{1}$ Laurie K. Bale, ${ }^{2}$ \\ Vicente E. Torres, ${ }^{3}$ Peter C. Harris, ${ }^{3}$ Claus Oxvig, ${ }^{4}$ Cheryl A. Conover, ${ }^{2}$ and Eduardo N. Chini ${ }^{1}$ \\ 'Department of Anesthesiology and Robert and Arlene Kogod Center on Aging, 'Division of Endocrinology and Metabolism, \\ Endocrine Research Unit, Mayo Clinic, Rochester, Minnesota, USA. ${ }^{3}$ Division of Nephrology and Hypertension and Robert \\ M. and Billie Kelley Pirnie Translational PKD Center, Rochester, Minnesota, USA. ${ }^{4}$ Department of Molecular Biology and \\ Genetics, Aarhus University, Aarhus, Denmark.
}

Autosomal dominant polycystic kidney disease (ADPKD) is the most common genetic cause of end-stage renal disease (ESRD). The treatment options for ADPKD are limited. We observed an upregulation in several ICF-1 pathway genes in the kidney of $P k d 1^{R C / R C}$ mice, a model of ADPKD. Pregnancy-associated plasma protein A (PAPP-A), a metalloproteinase that cleaves inhibitory IGF binding proteins (IGFBPs), increasing the local bioactivity of IGF-1, was highly induced in the kidney of ADPKD mice. PAPP-A levels were high in cystic fluid and kidneys of humans with ADPKD. Our studies further showed that PAPP-A transcription in ADPKD was mainly regulated through the CAMP/CREB/CBP/p300 pathway. Pappa deficiency effectively inhibited the development of cysts in the $P k d 1^{R C / R C}$ mice. The role of PAPP-A in cystic disease appears to be regulation of the IGF-1 pathway and cellular proliferation in the kidney. Finally, preclinical studies demonstrated that treatment with a monoclonal antibody that blocks the proteolytic activity of PAPP-A against ICFBP4 ameliorated ADPKD cystic disease in vivo in $P k d 1^{R C / R C}$ mice and ex vivo in embryonic kidneys. These data indicated that the PAPP-A/IGF-1 pathway plays an important role in the growth and expansion of cysts in ADPKD. Our findings introduce a therapeutic strategy for ADPKD that involves the inhibition of PAPP-A.

Authorship note: SK and KZH contributed equally to this work.

Conflict of interest: ENC has applied for a US patent on materials and methods for treating polycystic kidney disease (application 62/822,602).

Copyright: (c) 2020, American Society for Clinical Investigation.

Submitted: December 12, 2019

Accepted: January 22, 2020

Published: February 27, 2020

Reference information: $/ \mathrm{Cl}$ Insight 2020;5(4):e135700.

https://doi.org/10.1172/jci.

insight. 135700 .

\section{Introduction}

Autosomal dominant polycystic kidney disease (ADPKD) is a chronic kidney disease characterized by fluid-filled cysts in renal tubules. Progressive growth and expansion of these cysts often leads to end-stage renal disease (ESRD). It is caused mainly by mutations in PKD1 or PKD2, which encode polycystin-1 (PC-1) and PC-2, respectively (1). This is a systemic disorder that is characterized primarily by the bilateral formation of fluid-filled renal cysts, as well as extrarenal manifestations. Progressive enlargement of renal cysts over time leads to chronic renal injury and often renal failure. ADPKD is responsible for $5 \%-10 \%$ of ESRD cases $(2,3)$. Unfortunately, treatment options for ADPKD are very limited (4). A better understanding of the pathophysiology of ADPKD is necessary for the development of more effective therapies for the management of this systemic disease.

Cystogenesis in ADPKD has been proposed to include 2 phases, cyst initiation and cyst expansion. The renal cysts in ADPKD originate from nephrons and the renal collecting tubule system, and their expansion involves fluid secretion and hyperproliferation of renal tubular epithelial cells (5). Cyst initiation appears to be mediated by loss or reduction of function of PC-1 or PC-2. The activity of the polycystin complex is believed to be essential for preventing cell-autonomous renal epithelial cell cytogenesis. The subsequent expansion of cysts is a key component of the pathogenesis of ADPKD. Cyst expansion compresses and damages tissue, causing inflammation and fibrosis, and eventually resulting in kidney failure (6). Several signaling molecules, including CAMP, mTOR, AMPK, and growth factors have been implicated in the pathogenesis of ADPKD, and many of these pathways have been shown to be modulated by the energy state of the organism (6-9). Recent findings have revealed that metabolic alterations (10) including defective glucose metabolism (7, 11-13), impaired beta-oxidation (14), and abnormal mitochondrial activity (15-17) play a role in ADPKD pathogenesis. In fact, recent studies demonstrated that dietary intervention 
and metabolic reprogramming are effective approaches for slowing cyst expansion and disease progression in murine models of $\operatorname{ADPKD}(7,18)$. In particular, we and others have demonstrated the protective effects of mild to moderate calorie/food restriction (FR) (10\%-40\% decrease in total caloric intake) in multiple murine models of $\operatorname{ADPKD}(18,19)$. In addition to that, a recent study reported that time-restricted feeding and ketosis led to protective effects against ADPKD in rat, mouse, and feline models of ADPKD (20).

FR without malnutrition extends lifespan and increases resistance to age-related diseases in rodents and monkeys, improves the health of overweight humans, and has recently been reported to delay the progression of $\operatorname{ADPKD}(8,9,18,19,21,22)$. Interestingly, the beneficial effects of FR in aging and life span appear to be mediated in large part by suppression of the IGF pathway $(8,9,21,22)$. IGF signaling plays an important role in several physiological and pathological conditions, including aging, cancer, and tissue growth (23-26). IGF pathway dysregulation has been implicated in kidney diseases (27). It has also been speculated that IGF-1 may play a role in ADPKD, though support for this theory in the literature is correlative and limited, e.g., elevated expression of IGF-1 has been reported in ADPKD patients (28) as well as animal models $(18,29,30)$. Additionally, IGF-1 has been shown to promote in vitro proliferation of renal cystic epithelial cells derived from ADPKD patients $(29,31,32)$. While these observations are suggestive of a potential role for the IGF pathway in the pathogenesis of ADPKD, this hypothesis remains largely untested.

Under physiological conditions, circulating IGF-1 is bound to IGF binding proteins (IGFBPs) with high affinity, which increases its half-life in tissues; the IGFBPs prevent interaction of IGF-1 with its cell surface receptors, limiting its bioavailability and cellular effects (33). Pregnancy-associated plasma protein A (PAPP-A) is a secreted and cell-associated metalloproteinase that cleaves IGFBP4 in an IGF-dependent manner, freeing IGF-1 for receptor binding and activation $(34,35)$. In the present study, we identified a direct role for PAPP-A in the pathogenesis of ADPKD. Cystic fluid and kidneys from ADPKD patients showed a significant increase in PAPP-A expression. Similarly, a significant increase in PAPP-A levels was observed in ADPKD cells in vitro and in murine models of ADPKD, in the latter correlating positively with disease severity. Both genetic ablation and pharmacological inhibition of PAPP-A conferred remarkable protection against cystic disease, demonstrating that the PAPP-A/IGF signaling pathway plays a crucial role in ADPKD pathogenesis. Consequently, targeting of PAPP-A could be a novel therapeutic approach to treat this disease.

\section{Results}

PAPP-A levels increase with the progression of cystic disease. Despite speculation that the IGF-1 pathway may be involved in the pathogenesis of $\operatorname{ADPKD}(18,28,29)$, no in vivo studies to our knowledge have been performed to directly investigate this hypothesis. The IGF pathway is complex and has several components including IGF ligands, IGFBPs, the IGF receptor, and IGFBP-cleaving enzymes such as pappalysins (PAPP-A and PAPP-A2) (33-35). As a first step, we evaluated the renal expression of components of the IGF pathway in the $P k d 1^{R C / R C}$ murine model of $\mathrm{ADPKD}$ (18). Using real-time PCR (RT-PCR), we observed upregulation of several IGF pathway genes in kidney tissues of the $P k d 1^{R C / R C}$ mice, including $\operatorname{Ig} f 1$, Igflr, and $\operatorname{Igfbp5}$. The greatest induction, approximately 8-fold, was observed with Pappa (Figure 1A), which increases IGF-1 bioavailability through cleavage of ligand-bound IGFBP4. IGFBP5 expression has been shown to be induced by the activation of IGF-1 availability and is considered a positive in vivo marker of IGF-1 signaling (36-38).

We thus hypothesized that increased PAPP-A expression may play an active role in the pathogenesis and progression of ADPKD. To initially explore this possibility, we determined whether Pappa mRNA expression correlated with pathological parameters such as kidney size and cystic index. A near-perfect positive correlation, with $r^{2}=0.9$, was observed between Pappa mRNA and kidney/heart weight ratio (Figure 1B). In addition, strong positive correlation existed between PAPP-A expression or kidney/heart weight ratio and markers of renal injury and fibrosis during first 7.5 months of the disease (Supplemental Figure 1, A-C; supplemental material available online with this article; https://doi.org/10.1172/jci. insight.135700DS1). These data suggest that Pappa expression is concomitant with the progression of cystic disease, and may be directly associated with the growth and expansion of the cysts in ADPKD at a threshold that correlates with tissues injury, inflammation, and fibrosis.

PAPP-A plays an important role in various biological processes such as the normal healing response and healthy ovarian follicular development, and regulates prenatal or postnatal growth and skeletal muscle formation (39-42). PAPP-A is also involved in pathogenesis of several disease and is a therapeutic target in diseases such as atherosclerosis and cancer, as well as age-related diseases (43-48). Plasma PAPP-A has 
A
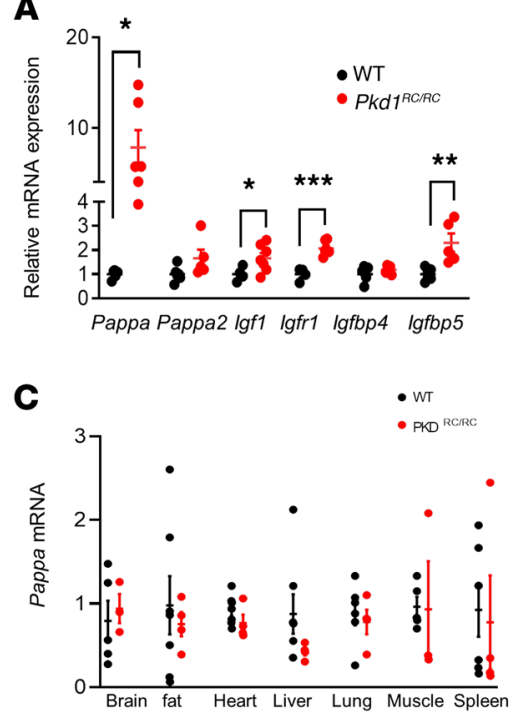

B

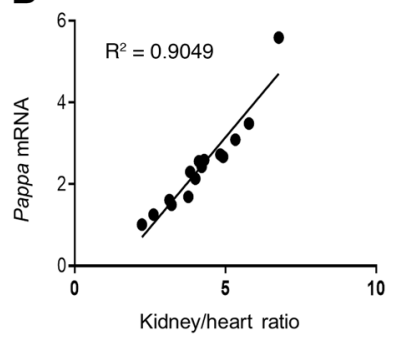

D

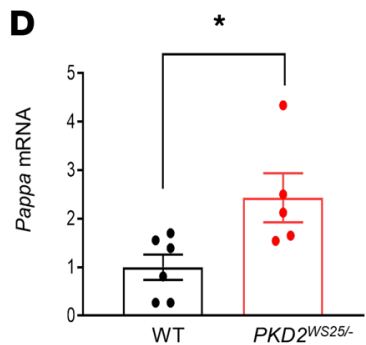

E

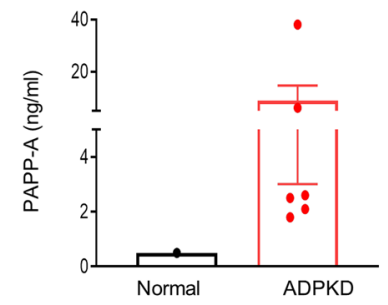

G

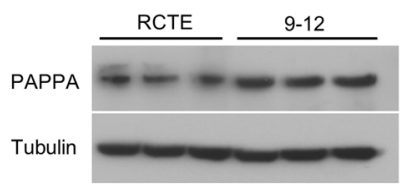

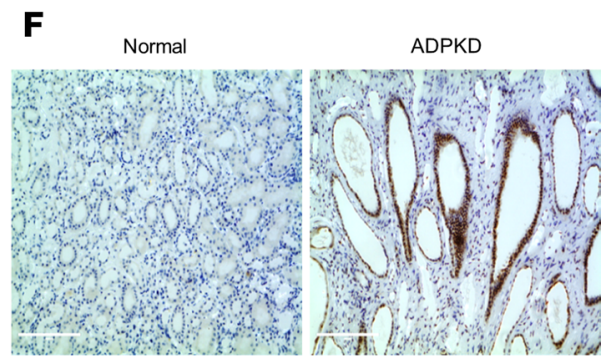

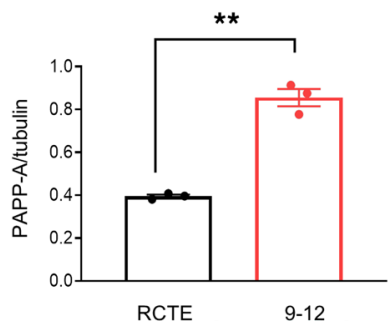

Figure 1. Upregulation of PAPP-A is a common feature in experimental and human ADPKD. (A) Relative mRNA expression of IGF-1 pathway components in kidneys of 7.5-month-old C57BL/6) $(n=4-6)$ and $P k d 7^{R C / R C}$ mice $(n=5-7)$. PCR data are expressed relative to Gapdh. (B) Correlation between kidney size (total kidney weight relative to heart weight) and renal Pappa mRNA expression in Pkd7RC/RC mice $(n=15)$. (C) Pappa mRNA levels in various tissues of WT $(n=3-5)$ and $P k d 7 R^{R / R C}$ mice $(n=4-6)$. (D) Pappa mRNA levels in WT $(n=6)$ and $P k d 2^{\text {WS25/- }}(n=5)$ mouse kidneys ( 16 weeks old). (E) ELISA analysis of PAPP-A protein levels in human ADPKD cystic fluid $(n=6)$ compared with normal serum reference. (F) Immunolocalization of PAPP-A in normal and ADPKD human kidneys. (C) Western blot analysis of PAPP-A protein levels in normal human RCTE and ADPKD cystic epithelial cells (9-12); graph shows quantification relative to tubulin. Scale bars: $200 \mu \mathrm{m}$. Data are expressed as mean \pm SEM. ${ }^{*} P<0.05,{ }^{* *} P<0.01,{ }^{* * *} P<0.001$ by 2 -tailed (for Igf1, 1-tailed) Student's $t$ test.

also been shown to correlate with renal function, been present at higher levels in patients on dialysis, and serve as an independent predictor of mortality of patients on hemodialysis (49-51). PAPP-A is ubiquitously expressed in several organs in humans $(41,52-56)$ and highly expressed in the human placenta (57). To examine whether the increase in Pappa expression is specific to the kidney in ADPKD, we compared Pappa mRNA levels in several tissues of 7.5-month-old WT and $P k d 1^{\mathrm{RC} / \mathrm{RC}}$ mice, including brain, lung, heart, liver, spleen, muscle, and fat. Interestingly, Pappa mRNA levels were elevated only in kidneys but not in other organs of $P k d 1^{R C / R C}$ mice (Figure 1, A and C), suggesting that the PAPP-A production is increased selectively in ADPKD kidneys. This observation further supports the idea that in ADPKD, augmented PAPP-A expression might cause increased cleavage of IGFBPs and hence increased availability of free IGF-1 to bind to its receptor. We hypothesize that this specifically occurs in the kidney, promoting ADPKD-related 
cellular proliferation and tissue growth. Interestingly we found that FR, which slowed cyst progression in $P k d 1^{R C / R C}$ mice, also decreased renal Pappa expression to normal levels (Supplemental Figure 1D). This further strengthens the hypothesis that PAPP-A may play a key role in pathogenesis of ADPKD.

Next, to determine whether the increase in PAPP-A levels is a common feature of ADPKD, we measured Pappa mRNA expression levels in a second murine model of ADPKD, $P k d 2^{W S 25 /-}$ mice. We found that, as in the $P k d 1^{R C / R C}$ mice, Pappa expression was also increased in the kidney of $P k d 2^{\mathrm{WS} 25 /-}$ mice, suggesting that PAPP-A could be a component of the pathogenesis of ADPKD in both murine models (Figure 1D).

We further investigated whether PAPP-A is increased in humans with ADPKD. Renal cystic fluid from ADPKD patients was assessed for PAPP-A using an ELISA, and the level of PAPP-A in cystic fluid was about 9 times higher than in serum, suggesting local synthesis and secretion into the cyst fluid (Figure 1E). Next, paraffin-embedded kidney sections from ADPKD patients and normal controls were probed with a polyclonal antibody against PAPP-A to determine the anatomical localization of expression. PAPP-A was found to be highly expressed on cystic epithelia and renal tubules of ADPKD patients (Figure 1F). In contrast, normal kidney sections showed diffused, low-level expression of PAPP-A (Figure 1F). In vitro experiments with human ADPKD cystic epithelial cells (designated "9-12" cells) derived from ADPKD patients (58) also demonstrated higher PAPP-A protein expression than normal human renal cortical tubular epithelial (RCTE) cells (Figure 1G). These findings indicate that PAPP-A is produced in the kidney in human ADPKD as well, is present in cystic epithelia, and is secreted into the cystic fluid.

The cAMP pathway induces PAPP-A expression in ADPKD. While the above findings of increased PAPP-A expression in ADPKD suggest its important role in the pathogenesis of this cystic disease, little is known about the mechanisms that regulate PAPP-A expression in the kidney. We therefore explored potential pathways that could regulate expression of PAPP-A in ADPKD.

PAPP-A expression has been shown to be regulated by proinflammatory cytokines, growth factors, as well as cAMP-inducing agents (35). In ADPKD, reduction of PC-1/PC-2 disrupt intracellular calcium homeostasis, resulting in a subsequent increase in intracellular cAMP levels in the kidney. cAMP has been shown to mediate cyst formation and promote fluid secretion, thus playing a central role in various signaling pathways leading to the pathogenesis of ADPKD (59-62). Interestingly, as mentioned above, in non-kidney cells, it has been shown that the cAMP pathway stimulates PAPP-A mRNA expression $(63,64)$. However, the precise mechanism by which cAMP stimulates PAPP-A expression has not been determined thus far.

To investigate whether cAMP plays a role in the regulation of PAPP-A expression in ADPKD, we first examined the effect of the cAMP-stimulating agent forskolin (FSK) on renal Pappa expression in vivo in $P k d 1^{R C / R C}$ and WT control mice. Pappa was not significantly induced until around 2.5 months age in $P k d 1^{R C / R C}$ mice, and its expression was highest around 7.5 months as the disease progressed; therefore, we treated the mice with FSK at 4 weeks of age, when Pappa mRNA expression levels were not elevated in $P k d 1^{R C / R C}$ mice (Supplemental Figure 2A). Twenty-four hours after injection with FSK (5 mg/ $\mathrm{kg}$ ) or DMSO, kidneys were isolated and gene expression was measured using RT-PCR. FSK induced a significant increase in renal Pappa mRNA expression in the $P k d 1^{R C / R C}$, but not WT, mice, while expression levels of $I g f b p 4, I g f b p 5$, and Igflr were similar in the 2 groups (Figure 2A and Supplemental Figure 2B). This indicates that cAMP could be a potent stimulator of the renal PAPP-A expression in ADPKD.

Using the 9-12 human ADPKD cells, we next examined the mechanism by which cAMP may induce PAPP-A expression in polycystic kidney disease (PKD). Upon FSK treatment, Pappa mRNA levels were highly induced in 9-12 cells compared with RCTE cells derived from normal kidney, which showed only a slight increase (Figure 2B). Furthermore, we found that FSK increased Pappa expression in a dose and time-dependent manner (Figure 2, B and C). We also assessed PAPP-A protein levels by ELISA in cellfree conditioned media of RCTE and 9-12 cells with and without FSK stimulation, and observed an 8-fold increase in 9-12 cells stimulated with FSK (Figure 2D), whereas RCTE cells showed a negligible increase. Further analysis of the cell-free conditioned media by measurement of PAPP-A-mediated IGFBP4 proteolysis demonstrated cleavage of IGFBP4 only in supernatant from FSK-stimulated 9-12 cells, indicating that proteolytically active PAPP-A is secreted from 9-12 cells only (Figure 2E). Addition of an inhibitory PAPP-A monoclonal antibody (65) blocked proteolytic cleavage of IGFBP4 (Figure 2E).

CAMP acts by activating at least 2 distinct cAMP-sensitive pathways: the PKA and the exchange protein directly activated by cAMP (Epac) pathways $(66,67)$. To determine whether PAPP-A expression in 9-12 cells is activated by the cAMP-PKA and/or cAMP-Epac pathway, we compared the effect of a selective PKA 
activator, 6-MB-cAMP (68), and a selective Epac activator, 8-CPT-2Me-cAMP (8CPT) (69), with that of the nonspecific cAMP agonist FSK. After 16 hours of treatment, 6-MB-cAMP caused the greatest increase in PAPP-A expression in PKD cells, which was somewhat higher than that in cells treated with FSK (Figure $2 \mathrm{~F}$ ). In contrast, only a small increase in PAPP-A expression was observed in 8CPT-treated PKD cells (Figure $2 \mathrm{~F}$ ). The role of the PKA pathway in induction of PAPP-A was further assessed in 9-12 cells using the PKA inhibitor Rp-cAMP (70). We found that inhibition of PKA significantly decreased FSK-induced PAPP-A expression in the PKD cells (Figure 2G), suggesting that cAMP might induce PAPP-A expression through activation of PKA. We also confirmed that relative PKA activity was similar in RCTE and 9-12 cells after induction with FSK (Supplemental Figure 2C).

Given that the in vitro data suggested a role for the PKA pathway in regulation of PAPP-A in ADPKD, we further examined this possibility in vivo using the $P k d 1^{R C / R C}$ mouse model with kidney-specific overactivation of PKA (71). These mice have a collecting duct-specific (Pkhd1-Cre) deletion (72) of PKA regulatory subunit 1a (Prkarla), whose function is to block PKA catalytic activity. At just 5 weeks, the burden of cystic disease in these mice was clearly increased compared with that in $P k d 1^{R C / R C}$ mice with normal PKA activity at 3 months (Supplemental Figure 2D), and a significant increase in PAPP-A expression was observed in them compared with $P k d 1^{R C / R C}$ mice at 3 months (Supplemental Figure 2E), confirming in vivo a role for PKA in the regulation of PAPP-A expression in ADPKD.

The cAMP signaling pathway activates the transcription factor cAMP response element-binding protein (CREB) through PKA. To study the involvement of CREB in cAMP-dependent PAPP-A expression in ADPKD, we treated PKD cells with KG-501, which attenuates cAMP-dependent gene induction by interacting with the CREB binding groove, which is necessary for CREB-CREB-binding protein (CREBCBP) interaction (73). KG-501 indeed decreased cAMP-induced PAPP-A expression in a dose-dependent manner, which suggested that CREB is involved in PAPP-A regulation (Figure $2 \mathrm{H}$ ).

The CREB:CBP/p300 complex binds to gene promoters, inducing histone acetylation, which "relaxes" the chromatin and increases target gene expression (74). We next investigated whether the interaction between CREB and CBP/p300 activates PAPP-A transcription in ADPKD. CBP30, which preferentially binds to the $\mathrm{CBP} / \mathrm{p} 300$ bromodomain and blocks gene transcription (75), was used for this study. The results showed that CBP30 abolished FSK-induced PAPP-A expression in PKD cells (Figure 2I). To determine whether the CBP inhibition can also abolish PAPP-A expression in vivo, we treated $P K D 1^{R C / R C}$ mice with the $30 \mathrm{mg} / \mathrm{kg} \mathrm{CBP}$ inhibitor GNE-049 twice a day for 3 days orally (76). We found a significant reduction in PAPP-A expression in mice treated with CBP inhibitor compared with the vehicle-treated mice (Figure 2J). Thus, it can be concluded that the CAMP-PKA/CREB/CBP/p300 pathway regulates PAPP-A expression in ADPKD (Figure $2 \mathrm{~K})$. The AMPK pathway has been shown to affect the IGF pathway (77) and also be involved in PKD; therefore, to determine whether AMPK activation has an effect on PAPP-A expression, we stimulated 9-12 cells with the AMPK stimulator A769662 (78). A769662 significantly reduced cAMP-induced Pappa expression in 9-12 cells (Supplemental Figure 2F). However, the AMPK inhibitor compound C was unable to increase cAMP-induced PAPP-A expression in RCTE cells (Supplemental Figure 2G).

In addition to cAMP, several other agents have also been shown to induce PAPP-A expression in non-kidney cells. In 9-12 and RCTE cells, we tested the effect on PAPP-A expression of a battery of agents that have either been shown to induce PAPP-A in other cell types or are known to play a role in the pathogenesis of ADPKD. There was a strong induction of PAPP-A expression only in 9-12 cells in response to FSK and a weak response to TGF- $\beta$ (Supplemental Figure 2H). Surprisingly, PAPP-A expression in 9-12 cells was not induced by any of the other agents tested, including EGF, IL-1 $\beta$, and IL-2 (Supplemental Figure 2H). Since ERK and calcium signaling pathways are involved in ADPKD pathogenesis, we tested whether ERK and calcium signaling could regulate PAPP-A expression in ADPKD. ERK inhibition in 9-12 cells using the ERK inhibitor SCH772984 (79) was unable to abolish cAMP-induced PAPP-A expression (Supplemental Figure 2I). Similarly, ionomycin treatment in 9-12 cells (Supplemental Figure 2J) and verapamil in RCTE cells (Supplemental Figure 2K) were unable to reduce or increase cAMP-induced PAPP-A expression, respectively, indicating that calcium signaling might not be involved in the regulation of PAPP-A expression. PAPP-A expression has been shown to be regulated epigenetically (80); therefore, we next tested whether PAPP-A expression could be epigenetically regulated in ADPKD cells. We assessed the effects of the hypermethylating agent budesonide and the hypomethylating agent decitabine on PAPP-A expression. Although budesonide significantly reduced cAMP-induced Pappa expression in 9-12 cells (Supplemental Figure 2L), decitabine did not induce Pappa expression in FSK treatment to the same extent in RCTE as in 9-12 cells (Supplemental Figure 2M). 

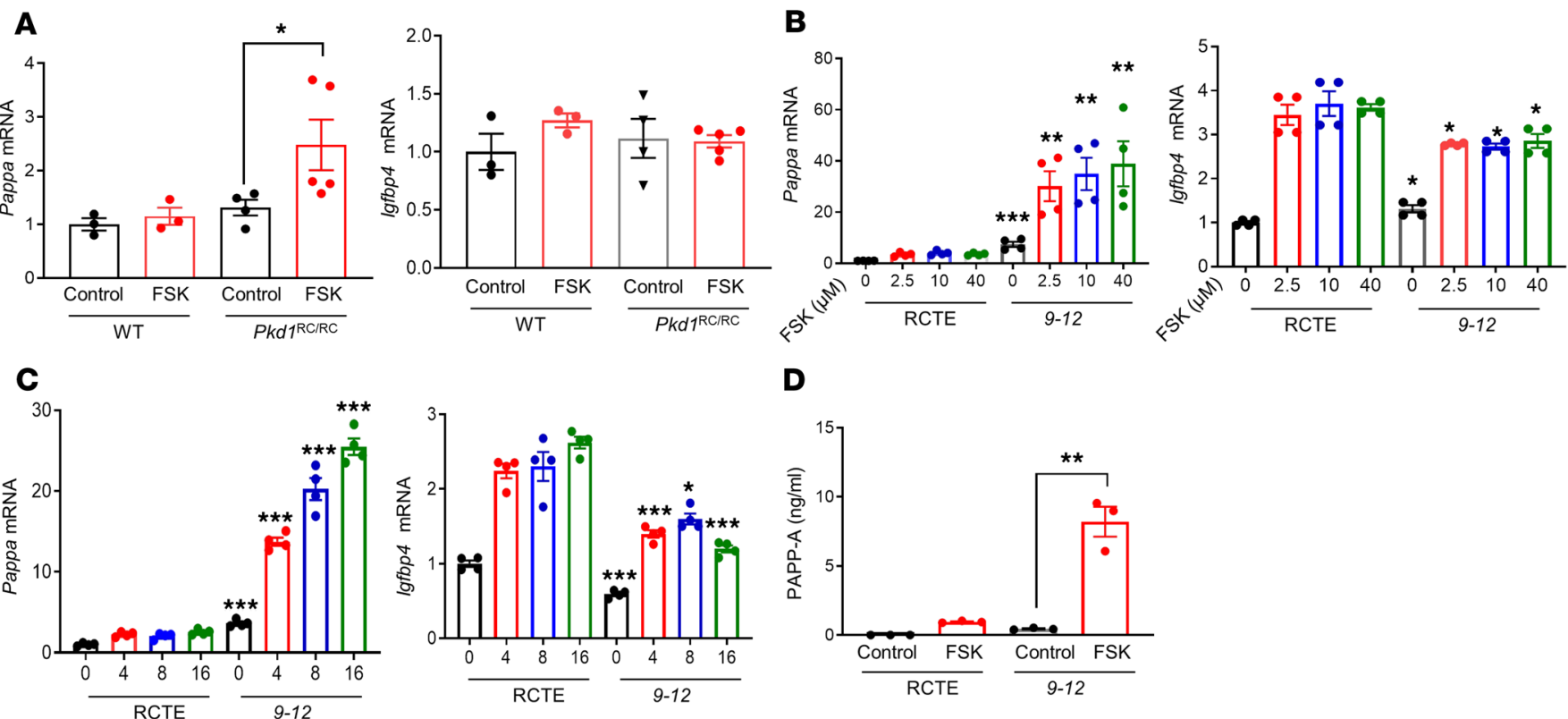

D

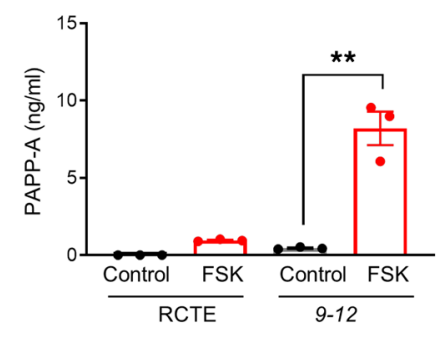

E

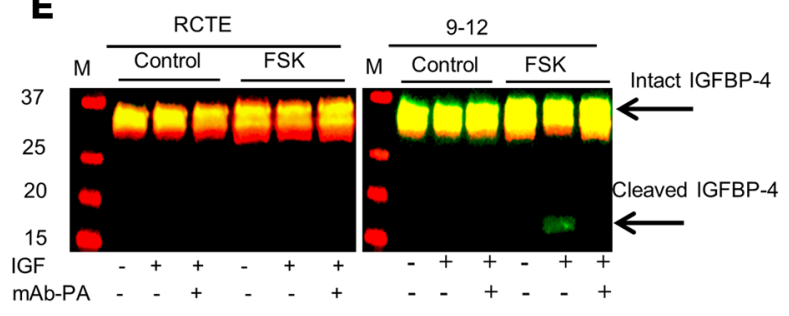

F
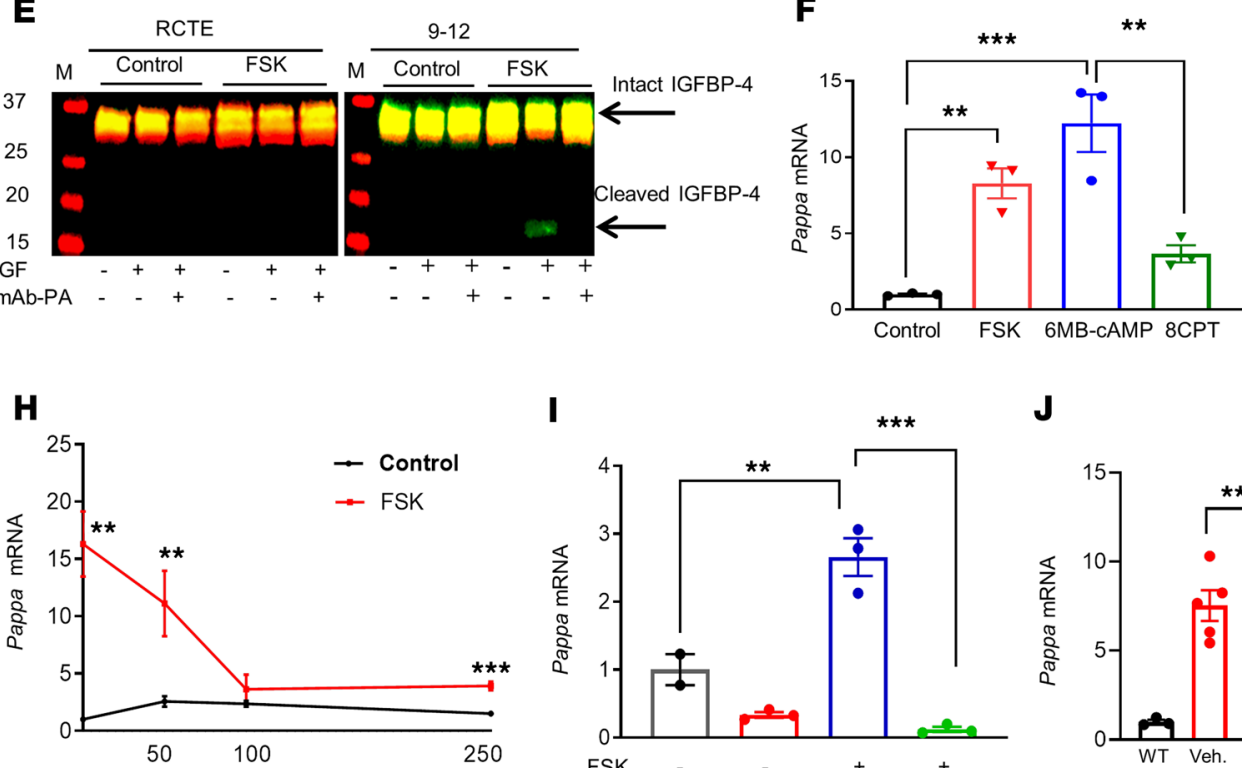

I

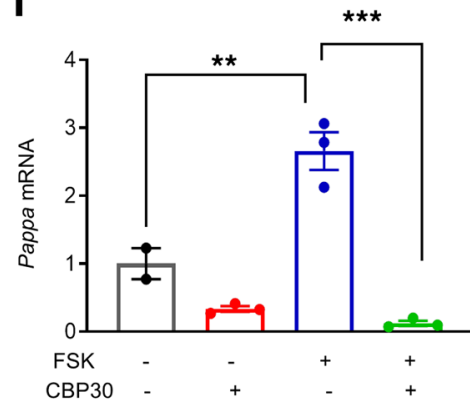

G

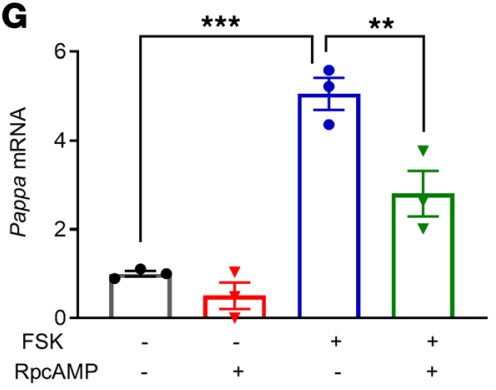

J

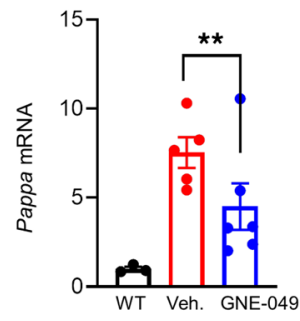

$\mathbf{K}$

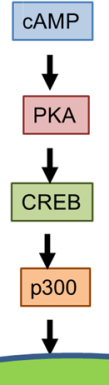

PAPP-A expression

Figure 2. Molecular pathways involved in regulation of PAPP-A expression. (A) Pappa and lgfbp4 mRNA levels in kidneys of $P k d 1 R C / R C$ ( $n=4-5)$ and WT mice $(n=3)$ treated with vehicle ( $5 \%$ DMSO) or $5 \mathrm{mg} / \mathrm{kg} \mathrm{FSK} \mathrm{for} 24$ hours at 4 weeks of age. (B and C) Pappa and Igfbp4 mRNA levels in RCTE and PKD cystic epithelial cells (9-12) treated with (B) increasing doses of FSK for 4 hours and (C) $10 \mu \mathrm{M}$ FSK for various time intervals. (D) ELISA analysis of PAPPA levels in cell-free conditioned media of RCTE and 9-12 cells treated with $10 \mu \mathrm{M}$ FSK or vehicle for 72 hours. (E) Proteolytic assay of PAPP-A-mediated IGFBP4 using cellfree conditioned media of RCTE and 9-12 cells treated with FSK or vehicle for 72 hours. Conditioned medium was incubated for 72 hours at $37^{\circ} \mathrm{C}$ with IGFBP4 without (-) or with (+) precomplexing to IGF, and without (-) or with (+) the addition of inhibitory mAb-PA 1/41 antibody. Arrows indicate intact and cleaved IGFBP4 bands. (F-I) Pappa mRNA expression in 9-12 cells treated with: (F) vehicle control (0.1\% DMSO), $10 \mu \mathrm{M}$ FSK, a selective activator of PKA (6-MB-cAMP, $200 \mu \mathrm{M}$ ) or Epac (8CPT20Me, $30 \mu \mathrm{M})$ for 16 hours; (C) $10 \mu \mathrm{M}$ FSK in the presence or absence of a competitive antagonist of cAMP (RpcAMP, $100 \mu \mathrm{M})$ for 16 hours; (H) $10 \mu$ M FSK in the presence or absence of the indicated doses of KG-501, which blocks cAMP-induction of CREB for 16 hours; (I) a selective CBP/p300 bromodomain inhibitor (CBP30, $10 \mu \mathrm{M}$ ) for 24 hours followed by $10 \mu \mathrm{M}$ FSK for 16 hours. (J) Pappa mRNA expression levels in WT and Pkd1 ${ }^{R C / R C}$ treated with the CBP inhibitor GNE-049 (30 mg/kg, $n=5$ ) or vehicle (Veh., $n=5$ ) twice a day for 3 days orally. (K) Schematic representation of cAMP-induced PAPP-A expression. Data are mean \pm SEM. ${ }^{*} P<0.05$, ${ }^{* *} P<0.01$, ${ }^{* *} P<0.001$ by 2 -tailed Student's $t$ test or Mann Whitney $U$ test, or 1-way ANOVA followed by Tukey's post hoc test (for $\mathbf{B}$ and $\mathbf{C}$, comparison of $9-12$ cells is at their respective dose or time with normal cells). 
Genetic deletion of Pappa in ADPKD mice remarkably reduces renal cystic burden, inflammation, fibrosis, and injury. To directly investigate the role of PAPP-A in the progression of cystic disease in ADPKD, we generated an ADPKD mouse model deficient in PAPP-A. These animals were generated by crossing the PAPP-A-deficient $P a p p a^{t m I C a c}$ mice (81) with the $P k d l^{R C / R C}$ mice (82). We observed that, like PAPP-A-deficient mice, $P k d 1^{R C / R C}$ Pappa $^{-/-}$(ADPKD-PAPP-A ${ }^{-/-}[\mathrm{KO}]$ ) mice were also smaller than their ADPKD-PAPP-A ${ }^{+/+}$and ADPKD$\mathrm{PAPP}_{-\mathrm{A}^{+/}}$littermates (Supplemental Figure $3 \mathrm{~A}$ ) but were overall healthy, with normal behavior. The progression of cystic disease in these mice was characterized at 3 different ages: 2.5, 4.5, and 12 months old. At each age, the kidneys of the ADPKD-PAPP-A KO mice were smaller and healthier in gross appearance compared with ADPKD-PAPP-A ${ }^{+/+}$mice (Figure 3A and Supplemental Figure 3B). A significant decrease in kidney weight was observed as assessed by kidney/body weight as well as kidney/heart weight ratios in ADPKD-PAPP-A KO mice compared with ADPKD-PAPPA ${ }^{+/+}$mice (Figure 3A and Supplemental Figure 3C). Remarkably, ADPKD-PAPP-A KO kidneys showed significantly reduced cyst area and, appeared like normal kidneys even at 12 months age as assessed by histological analysis (Figure 3B). Interestingly, ADPKD/PAPP-A ${ }^{+/-}$kidneys were also partially protected against cystic disease (Figure 3, A and B) and showed improvement in cystic burden (Figure 3B). We next assessed kidney function in these mice by measuring cystatin $\mathrm{C}$, the biomarker for renal function and glomerular filtration rate (GFR). Levels of cystatin C were significantly lower in the PAPP-A-deficient mice, and ADPKD-PAPP-A ${ }^{+/}$mice also showed an improvement (Figure 3C). Next, GFR was assessed in ADPKD-PAPP-A ${ }^{+/+}$and ADPKD-PAPP-A ${ }^{+/}$mice to overcome any confounding issue of smaller size in $\mathrm{KO}$ mice. Even one fewer copy of PAPP-A was able to significantly improve GFR compared with ADPKDPAPP-A ${ }^{+/+}$mice (Figure 3D). Both male and female PAPP-A-KO mice have been shown to live 30\%-40\% longer than the WT mice (45), and we found that $P k d 1^{R C / R C} \mathrm{Pappa}^{+/-}$(median survival, age 28 months) lived longer that $P k d 1^{R C / R C} \mathrm{Pappa}^{+/+}$mice (median survival, age 18 months) (Figure 3E). The sharp decline in survival of $P k d 1^{R C / R C} \mathrm{Pappa}^{+/+}$mice is compatible with death caused by kidney disease and not the aging process, since no age-related phenotype such as cataract or hair loss was observed in these mice.

Kidneys from ADPKD-PAPP-A mutants were further assessed for markers of inflammation, fibrosis, and renal injury. ADPKD-PAPP-A ${ }^{+/+}$kidneys had significantly higher levels of the inflammatory markers Mcp1 and Tnfa in comparison with age-matched WT control mice, whereas abrogation of PAPP-A significantly decreased renal inflammation in both ADPKD-PAPP-A ${ }^{-/}$and ADPKD-PAPP-A ${ }^{+/}$mice (Figure 4A). Staining of kidney tissues with anti-CD3 antibody also confirmed that PAPP-A deficiency reduced inflammation (Figure $4 \mathrm{~B}$ ).

Expression of neutrophil gelatinase-associated lipocalin ( $\mathrm{Ngal}$ ), a marker of both acute and chronic kidney injury, was analyzed to assess renal injury. We observed that in parallel with the development of kidney injury in the ADPKD animal model, Ngal mRNA expression was increased several-fold in ADPKD-PAPP-A ${ }^{+/+}$mice at both ages tested (4.5 and 12 months) compared with WT controls (Figure 4C). In contrast, decreased dosing of PAPP-A protected mice against kidney injury, as determined by Ngal mRNA levels (Figure 4C). Similarly, levels of the fibrosis markers collagen $1 \alpha 1$ (Colla1) and Tgfb were also reduced in ADPKD-PAPP-A ${ }^{-/}$and ADPKD-PAPP-A ${ }^{+/-}$mouse kidneys (Figure 4D). Fibrosis analysis by Sirius red staining further confirmed that PAPP-A deficiency was associated with reduced fibrosis (Figure 4E). In conclusion, abrogation of PAPP-A reduces cyst development, renal inflammation, injury, and fibrosis in a murine model of ADPKD.

Interestingly, cAMP levels were same in all $P k d 1^{R C / R C}$ Pappa mutants (Supplemental Figure 4A). Additionally, PAPP-A enzymatic activity in kidney in vivo was determined in ADPKD and KO mice. PAPP-A-mediated IGFBP4 proteolysis using kidney membrane fractions was assessed in WT, $P k d 1^{R C / R C}$, and $P k d 1^{R C / R C} \mathrm{Pappa}^{-1}$ mice. Cleavage of IGFBP4 was observed in membrane fractions of WT and $P k d 1^{R C / R C}$ mice but was negligible in Pappa-deficient mice, indicating that PAPP-A activity was higher in PKD mice but absent in $P k d 1^{R C / R C} \mathrm{Pappa}^{-1}$ mice (Supplemental Figure 4B). Importantly, ablation of PAPP-A had no significant effects on serum levels of total IGF-1 (Supplemental Figure 4C) or renal mRNA expression of Igfbp4, Igfr1 , and Igfl (Supplemental Figure 4D), indicating that PAPP-A promotes ADPKD cystic growth via degradation of IGFBP4, potentially increasing bioavailability of IGF to activate its receptor.

The role of PAPP-A in the pathogenesis of ADPKD: cellular proliferation and the IGF pathway. IGF-1 interacts with cell surface receptors, leading to activation of proliferation pathways such as Akt and ERK. It has previously been shown that the IGF-1 pathway can induce cellular proliferation in ADPKD-derived renal epithelial cells in vitro (31). To determine whether PAPP-A plays a role in regulation of IGF-1 pathways and activation of cellular proliferation, we first analyzed the activation of the IGF receptor IGFR1 and the 
A
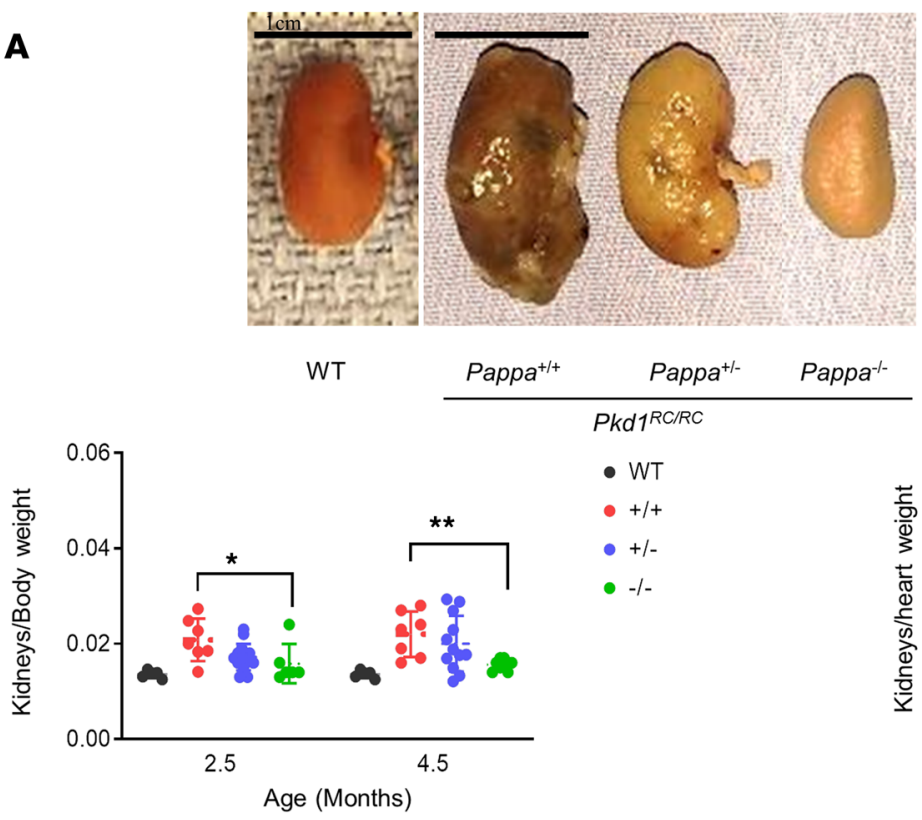

Pappa $^{+/-} \quad$ Pappa $^{-/-}$
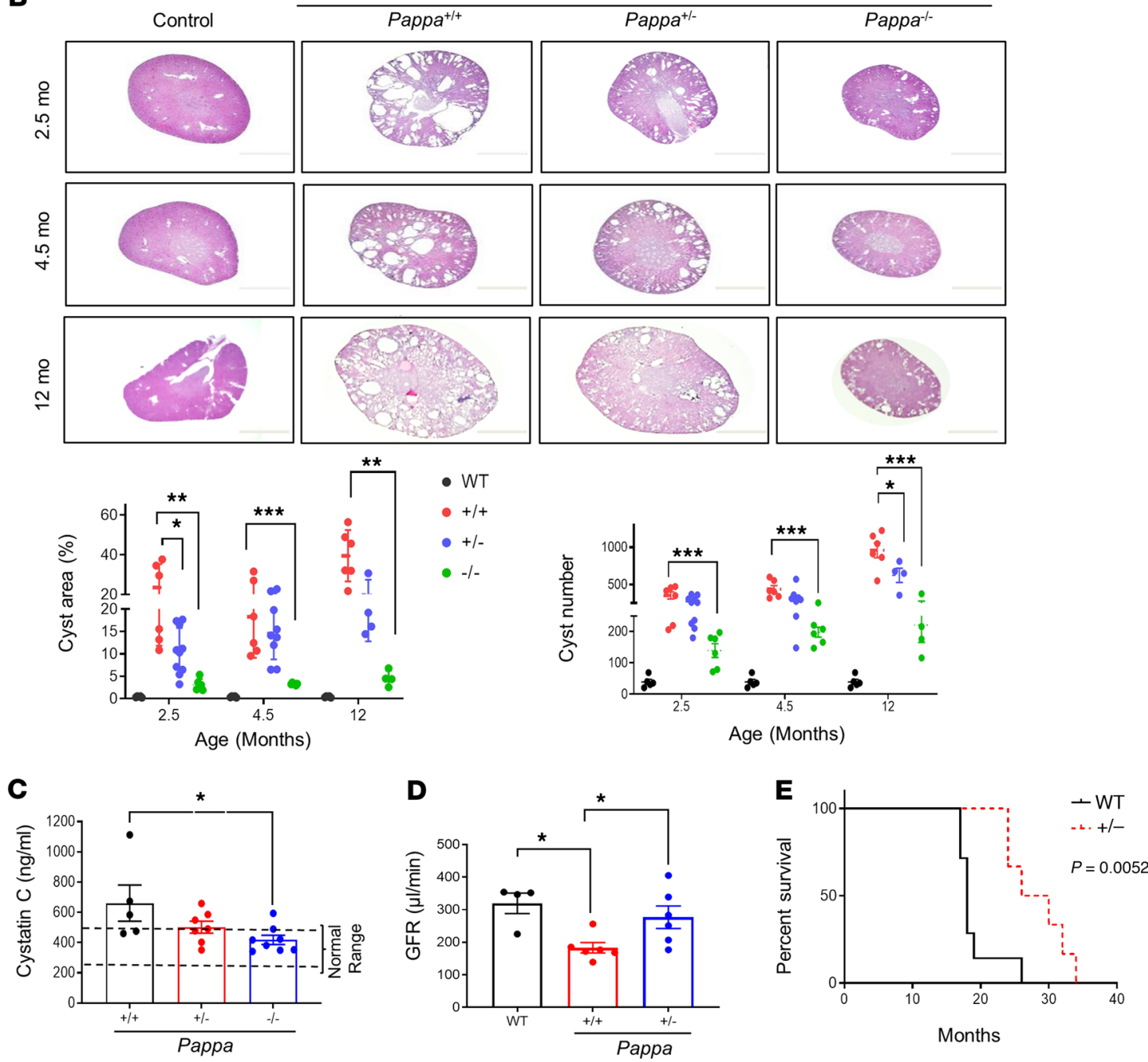
Figure 3. Genetic deletion of Pappa ameliorates ADPKD. (A and B) $P k d 1^{R C / R C}$ mice that are $\mathrm{Pappa}^{+/+}$, Pappa ${ }^{+/}$, or Pappa ${ }^{-/}$: (A) Representative gross kidney images at 12 month age, and graphs of kidney/body weight and kidney/heart weight at different ages $(n=5-15)$. Scale bars: $1 \mathrm{~cm}$. (B) H\&E kidney section photomicrographs and graphs of cystic area and cyst number compared with WT mice at different ages $(n=4-10)$. (C) Serum cystatin C levels in $P k d 7^{R C / R C}$ Pappa mutant mice at 12 months old age $(n=5-8)$. (D) GFR measurements in WT $(n=4), P k d 7^{R C / R C} \mathrm{Pappa}^{+/+}(n=6)$, and $P \mathrm{kd} 7^{R C / R C} P a p p a^{+/-}$mice $(n=6)$ at

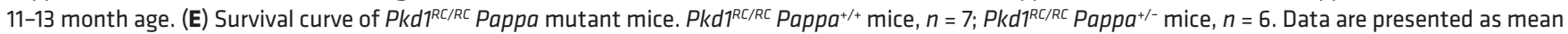
\pm SEM. ${ }^{*} P<0.05,{ }^{* *} P<0.01,{ }^{* *} P<0.001$ by 1 -way ANOVA followed by Tukey's post hoc test for parametric or Kruskal-Wallis test followed by Dunn's post hoc test for nonparametric distribution or 2-tailed Student's $t$ test.

markers of proliferation in kidney tissues of ADPKD-PAPP-A ${ }^{+/+}$and ADPKD-PAPP-A ${ }^{-/-}$mice by Western blot analysis. PAPP-A deficiency significantly reduced activation of IGFR1 (Figure 5A). Compatible with a role of PAPP-A as a regulator of the IGF-1 pathway and cellular proliferation in this disease, we observed that proliferating cell nuclear antigen (PCNA), a marker of cellular proliferation, was decreased in ADPKD-PAPP-A ${ }^{-/}$mice (Figure 5B). Interestingly, markers of neither autophagy (LC3 and p62) nor apoptosis (cleaved caspase-3) were different between ADPKD-APP-A ${ }^{+/+}$and ADPKD-PAPP-A ${ }^{-/}$animals (Supplemental Figure 5A). These data indicate that PAPP-A's role in ADPKD is primarily mediated by the regulation of cellular proliferative pathways. Thus, next we determined the role of PAPP-A in cellular signaling involved in the induction of cellular proliferation, such as the ERK and the Akt pathways. We observed that PAPP-A deficiency led to a decrease in phosphorylation of both ERK and Akt in animal models of ADPKD (Figure 5C). AMPK has been shown to suppress IGF-1 signaling (77); therefore, we next assessed expression of AMPK in ADPKD-PAPP-A ${ }^{+/+}$and ADPKD-PAPP-A ${ }^{-/}$mice and found that PAPP-A deficiency led to higher AMPK activation (Figure 5D). To further validate the role of the IGF1/IGFBP/PAPP-A pathway, we treated RCTE cells (which express low levels of PAPP-A) with IGF-1 alone, IGF-1 plus IGFBP4, or IGF-1 plus IGFBP4 and PAPP-A. We observed that IGF-1 alone induced activation of both ERK and Akt in these cells (Supplemental Figure 5B). This effect was blocked by preincubation of IGF-1 with IGFBP4 (Supplemental Figure 5B). However, addition of catalytically active PAPP-A recovered the levels of ERK phosphorylation in these cells (Supplemental Figure 5B), indicating that secreted PAPP-A cleaves IGF-1/IGFBP4, releasing free IGF-1 and allowing IGF-1 to activate downstream proliferation molecules such as ERK and Akt in renal cells.

As mentioned above, the role of the IGF-1 pathway has been speculated in ADPKD but no direct evidence exist. Therefore, to further investigate the role of IGF/IGFBP/PAPP-A pathway in the pathogenesis of ADPKD, we took advantage of the metanephric model of cyst induction (83). Using this model, first we explored whether IGF-1 has a permissive role in cyst formation in PKD. WT embryonic kidneys were stimulated with FSK alone in the absence of any growth hormone (insulin or IGF) or with FSK in the presence of IGF-1. Cystogenesis was observed only when both FSK and IGF-1 were present, suggesting that IGF-1 is necessary for FSK-induced cystic formation in this model (Figure 5E).

A three-dimensional Madin-Darby canine kidney (MDCK) cystogenic assay was also performed with FSK alone or in combination with IGF-1, and similarly indicated that IGF-1 in the presence of FSK significantly increases the number of cysts in these cells (Supplemental Figure 5C). Second, we assessed the effect of the IGF-IGFBP complex and PAPP-A on cyst induction in metanephros. Although FSK alone was not sufficient to induce cysts in this model, the addition of the IGF-1-IGFBP4 complex readily supported FSK-induced cystogenesis (Figure 5F). Interestingly, addition of a PAPP-A blocking antibody abrogated cyst formation in the embryonic metanephric model (Figure 5F). These data indicated that in the metanephric model endogenous PAPP-A can cleave IGFBP4 and increase the bioavailability of IGF-1 to activate its receptor and induce cystogenesis. To further explore this observation, we evaluated expression of PAPP-A in the metanephric model. Indeed, consistent with our hypothesis, we observed that FSK treatment led to a robust induction of PAPP-A expression in metanephros (Supplemental Figure 5D). Next, we determined whether endogenous levels of IGF1 in $P k d 1^{R C / R C}$ metanephric culture would promote cAMP-induced cystogenesis in the absence of any external growth hormone. Indeed, we found that cAMP induction alone (by FSK) was able to promote cyst formation in PKD metanephric kidneys even in the absence of external growth hormone (Supplemental Figure 5E).

Next, we determined the role of the IGF-1 pathway in ADPKD in vivo using $P k d 1^{R C / R C}$ mice. $P k d 1^{R C / R C}$ mice were injected with either an anti-IGF-1 antibody $(0.2 \mathrm{mg} / \mathrm{kg}$ body weight $)$ or $\operatorname{IgG}(0.2 \mathrm{mg} / \mathrm{kg}$ body weight $)$ once a week for 6 weeks. We observed an improvement in kidney weight, cystic burden, and molecular markers of inflammation, fibrosis, and kidney injury (Supplemental Figure 5, F and G). These results directly demonstrate for a role for the IGF-1 pathway in the pathogenesis of ADPKD (Supplemental Figure 5, F and G). 
A
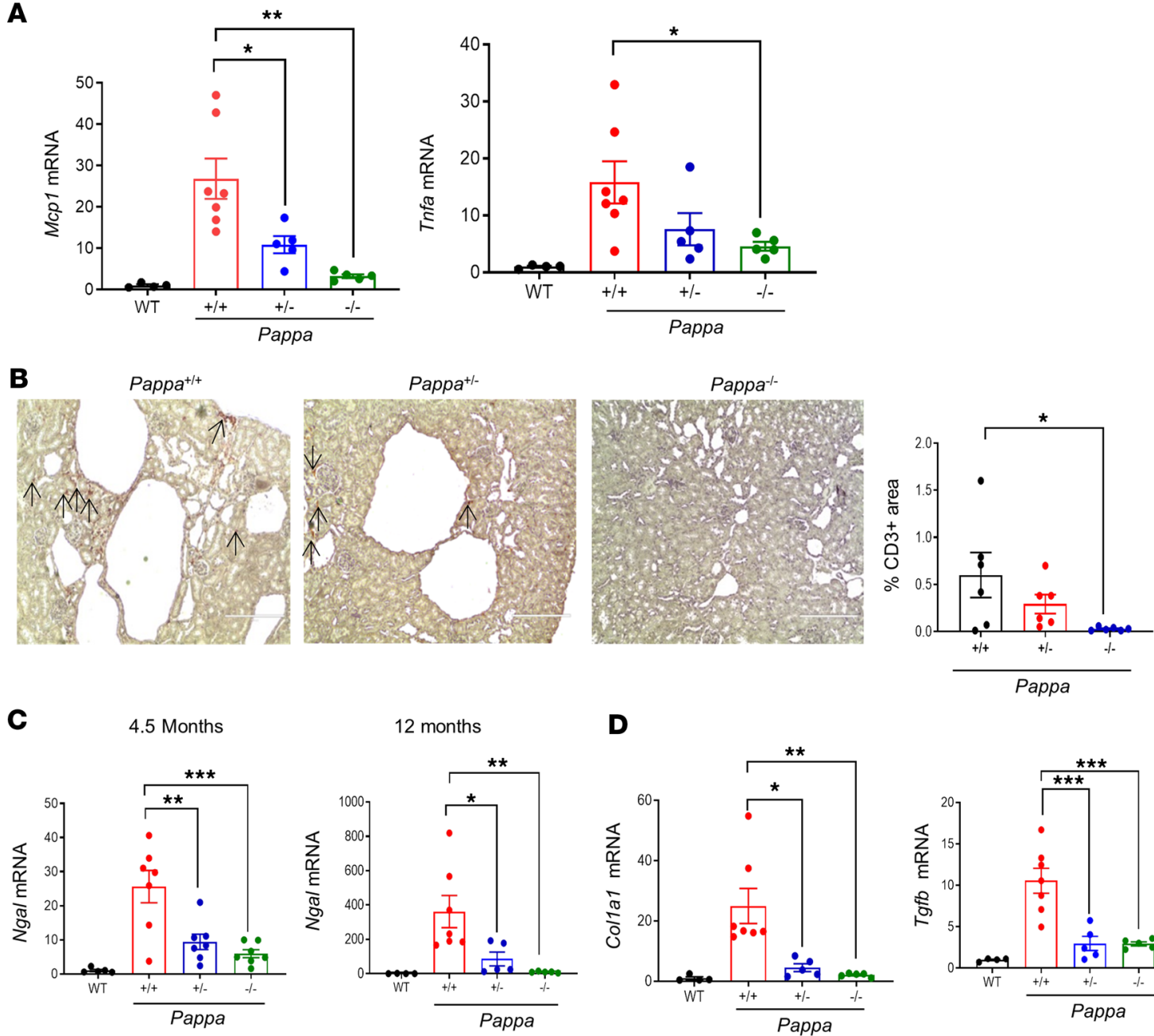

D
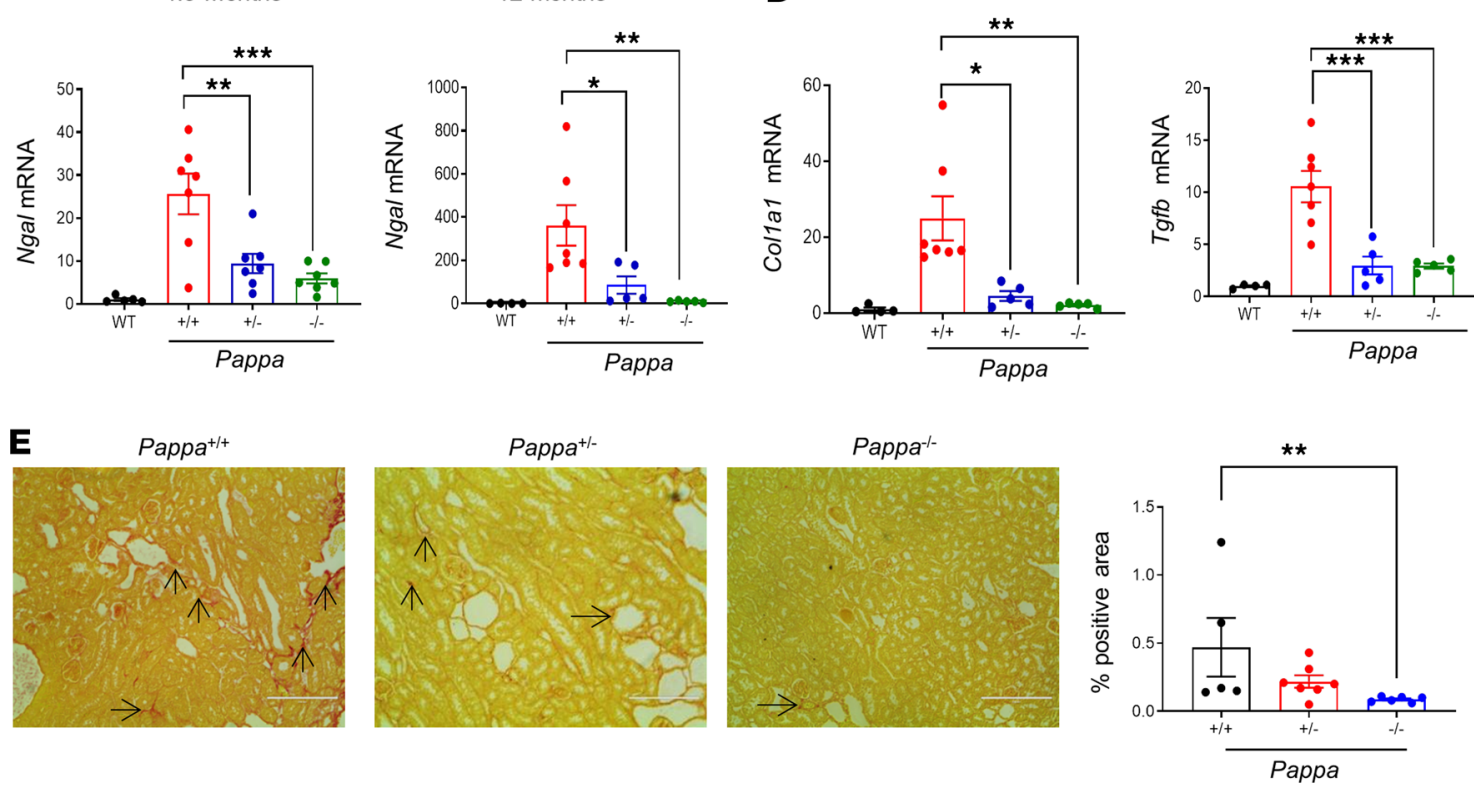

Figure 4. Genetic deletion of Pappa reduces renal inflammation, injury, and fibrosis in ADPKD mice. (A) Renal Mcp1 and Tnf $\alpha$ mRNA expression in WT and $P k d 1^{R C / R C}$ Pappa mutants $(n=4-7)$ at 12 months of age. (B) Representative photomicrographs showing anti-CD3 immunostaining in kidney sections from 4.5-month-old Pkd1 RC/RC Pappa mutant mice, and graph showing quantification of positively stained area ( $n=6 / g$ group). (C) Renal $\mathrm{Ngal}$ mRNA expression in WT and Pkd1RC/RC Pappa mutants at 4.5 and 12 months of age $(n=4-7)$. (D) Col1a 1 and Tgfb mRNA expression in WT and Pkd1 ${ }^{R C / R C}$ Pappa mutants $(n=4-7)$. (E) Representative photomicrographs of Sirius red staining in kidneys of 4.5-month-old PkdRT/RC Pappa mutant mice, and graph showing quantification of positively stained area $(n=5-7)$. Data are presented as mean $\pm \mathrm{SEM}$. ${ }^{*} P<0.05$, ${ }^{* *} P<0.01$ by 1 -way ANOVA and then Dunnett's post hoc test for parametric or Kruskal-Wallis test followed by Dunn's post hoc test for nonparametric distribution. Two-tailed Mann Whitney $U$ test was used for E. Original magnification, $\times 200$; arrows define the positively stained area.

Taken together, these data indicated that the IGF/IGFBP4/PAPP-A pathway plays an important role in the pathogenesis of ADPKD. In particular, PAPP-A via this pathway regulates the growth and expansion of cysts. Additionally, it indicates that targeting this pathway may have a therapeutic benefit for ADP$\mathrm{KD}$ patients. One of the advantages of targeting PAPP-A versus IGF-1R is that PAPP-A overexpression might be kidney specific in ADPKD.

In vivo inhibition of PAPP-A using mAb-PA blocks the progression of ADPKD. To investigate whether 
A

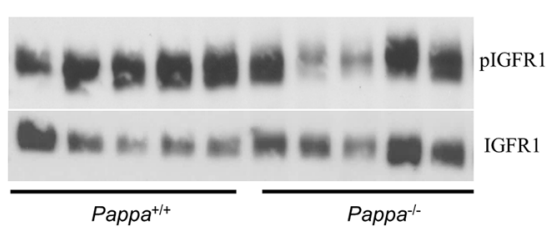

B

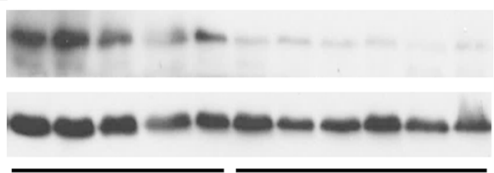

$\mathrm{Pappa}^{+/+}$
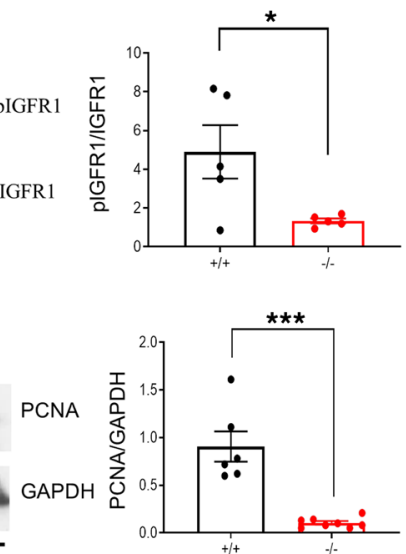

Pappa $^{-1-}$
E
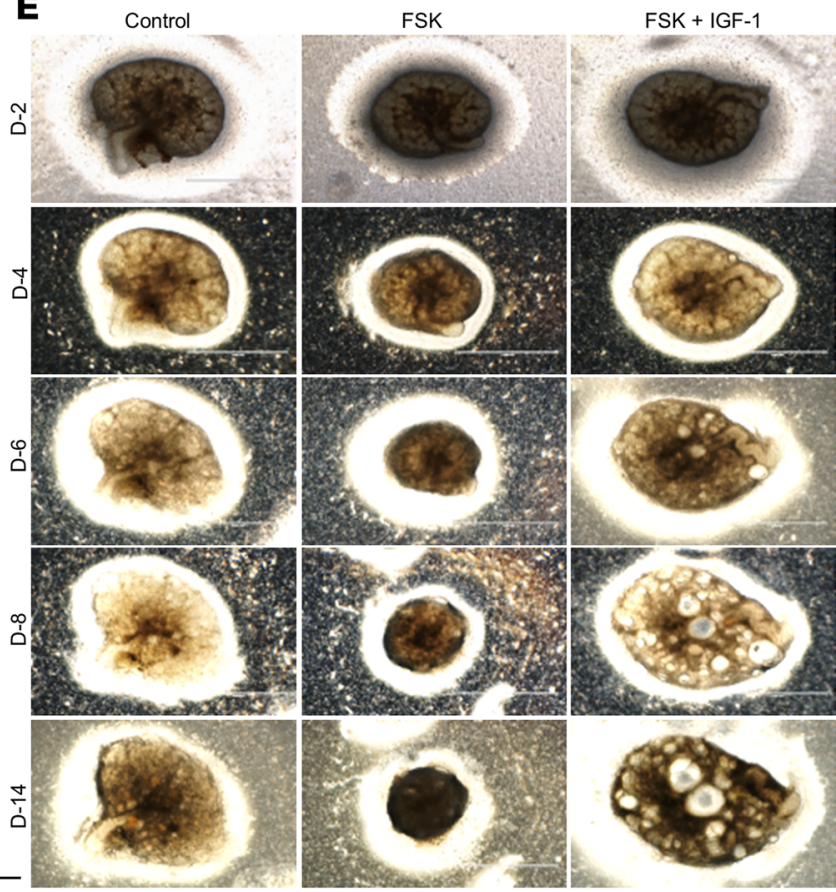

C
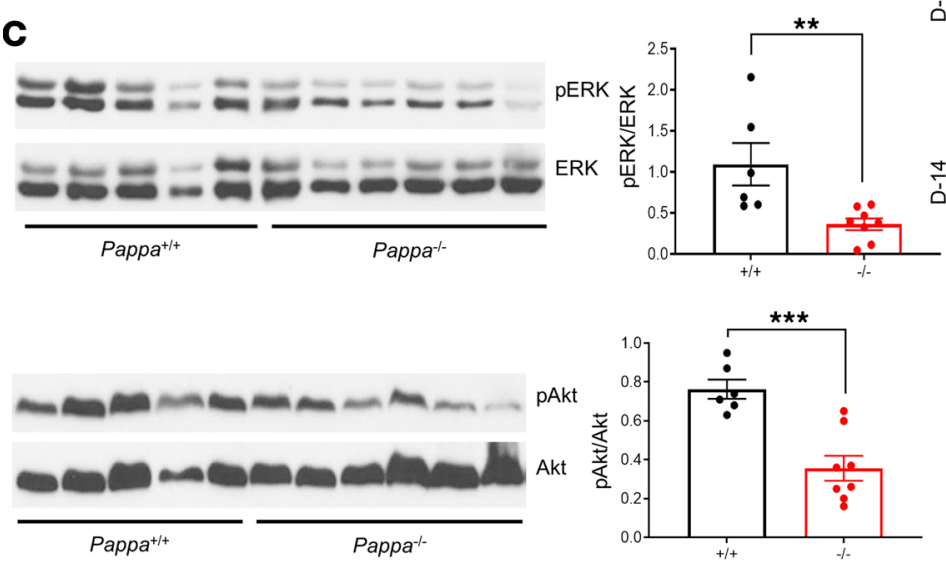

Figure 5. The role of PAPP-A in the pathogenesis of ADPKD.

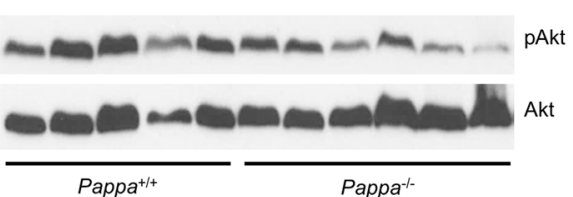

Western blot analysis of (A) p-ICFR1/IGFR1 (B) PCNA, (C) p-ERK/ERK and p-Akt/Akt, and (D) p-AMPK/AMPK in kidney tissues of 2.5- or 4.5-month-old $P$ kd $7^{R C / R C} \mathrm{Pappa}^{+/+}$and $P$ kd $1^{R C / R C} \mathrm{Pappa}^{-/-}$mice. Graphs show quantitative analysis of bands by densitometry. (E) Photomicrographs showing that ICF-1 supports cystic growth in a metanephric model of cystogenesis. Day 13.5 embryonic kidneys from WT mice were stimulated with FSK $(10 \mu \mathrm{M})$ alone (no growth hormone added) or with FSK in the presence of IGF-1 (100 ng/mL). Scale bars: 1

D

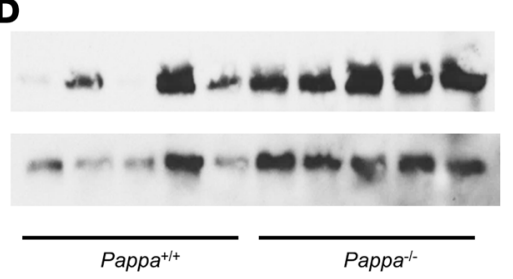

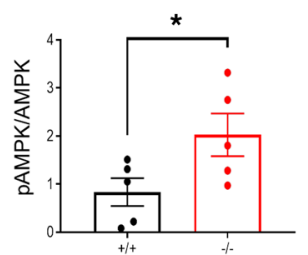
$\mathrm{mm}$. (F) Embryonic kidneys were treated with FSK (10 $\mu \mathrm{M})$, IGF-1 (100 $\mathrm{ng} / \mathrm{ml}$ ), and IGFBP4 (26 $\mathrm{nM}$ ) in the presence or absence of mAb-PA (320 pM). IGF-1 was incubated with IGFBP4 prior to treatment. For metanephric culture, the experiment was repeated twice; $n=3$ for each experiment. Data are mean $\pm \mathrm{SEM} .{ }^{*} P<0.05,{ }^{* *} P<0.01,{ }^{* * *} P<$ 0.001 by 2 -tailed Student's $t$ test (1-tailed for $\mathbf{D})$. Scale bars: $1 \mathrm{~mm}$.

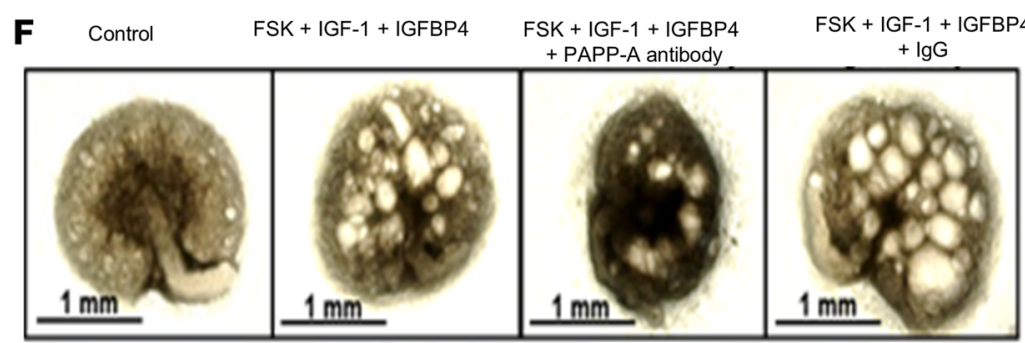

inhibition of PAPP-A ameliorates cystic disease, we used a high-affinity IgG monoclonal antibody against a substrate-binding exosite of PAPP-A (mAb-PA) that blocks the proteolytic action of PAPP-A on IGF-1/IGFBP4 $(65,81) . P k d 1^{R C / R C}$ mice were treated at 6 months of age with $30 \mathrm{mg} / \mathrm{kg} \mathrm{mAb}$ PA or IgG once a week i.p. for 6 weeks. All the antibody-treated mice were healthy, with normal behavior. The mAb-PA-treated mice showed a significant decrease in cystic burden, as assessed by reduced kidney size and cyst area compared with IgG-treated mice (Figure 6A). Although blood urea nitrogen (BUN) is not drastically increased in $P k d 1^{R C / R C}$ mice until a later age, we still observed significant decrease in BUN levels in the mice treated with mAb-PA (Figure 6B). Levels of the markers 
A
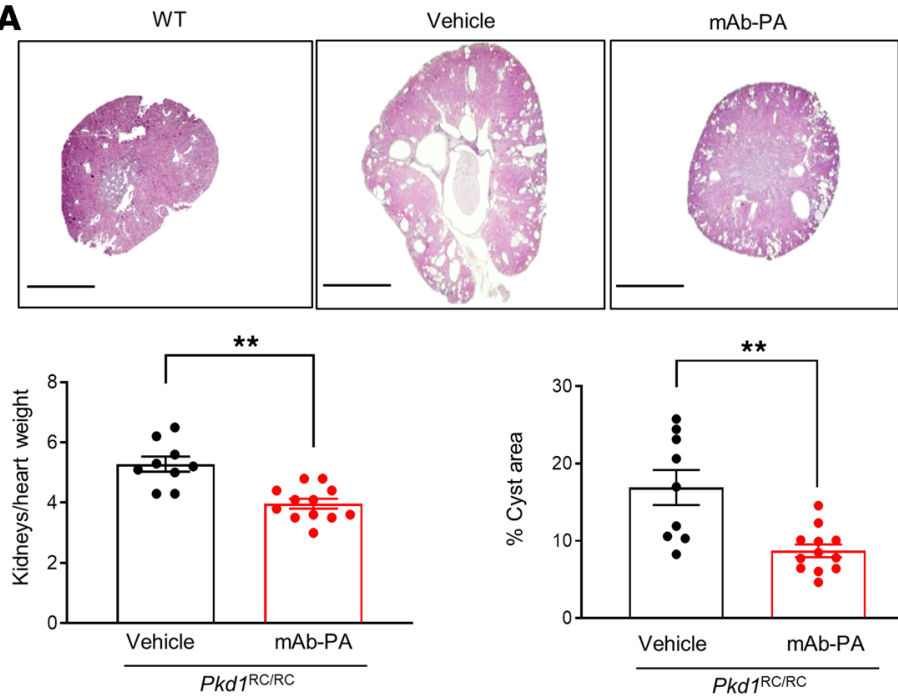

B
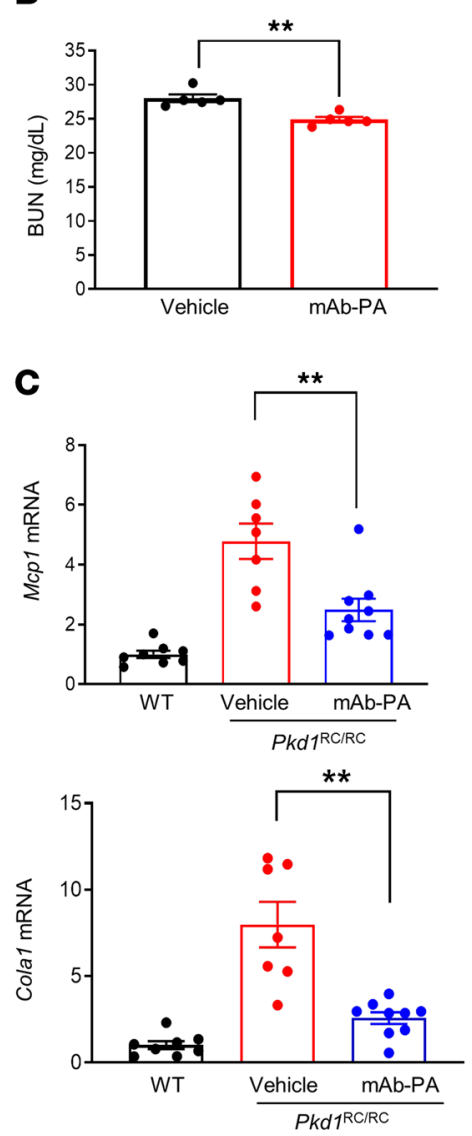
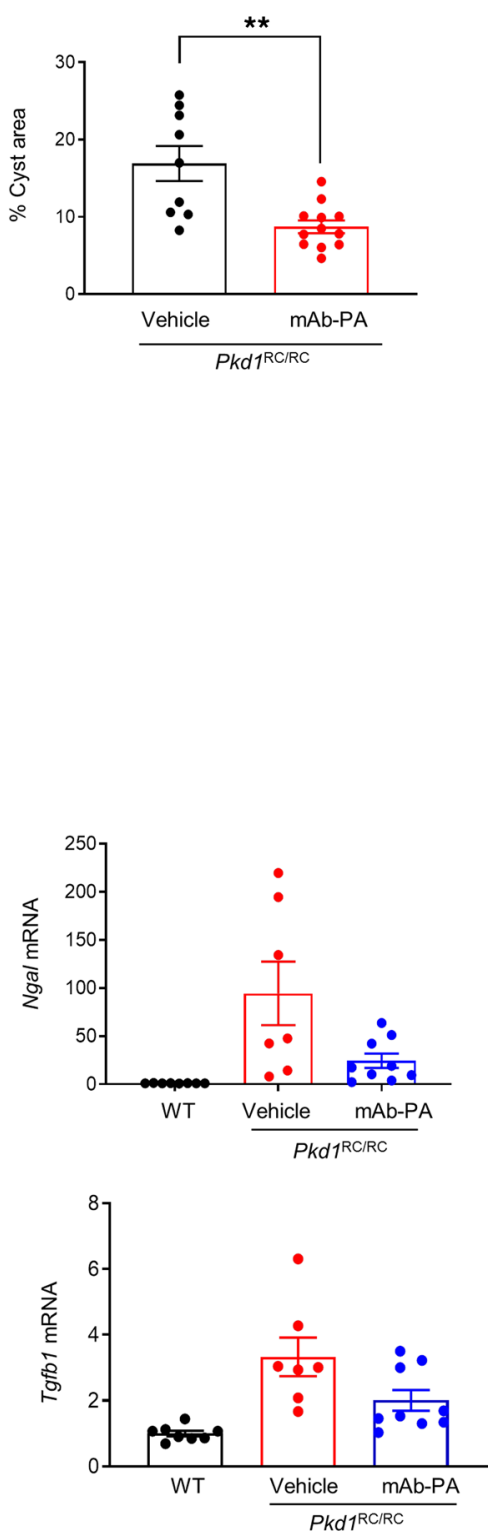

Figure 6. Blockade of PAPP-A function with monoclonal antibody (mAb-PA) decreases cyst formation in ADPKD mice. $P k d 1^{R C / R C}$ mice were treated with $\mathrm{mAb}-\mathrm{PA}(30 \mathrm{mg} / \mathrm{kg})$ or vehicle once per week for 6 weeks. (A) Representative images of H\&E-stained kidney sections and graphs showing kidney size and percent cystic area; age, 7.5 months $(n=9-12)$. Scale bars: $2000 \mu \mathrm{m}$. (B) BUN measurements in vehicle $(n=5)$ and anti-PAPP-A-treated mice $(n=5)$. (C) Mcp1, Ngal, Col1a1, and Tgfb mRNA expression in vehicle or mAb-PA-treated $P k d 1^{R C / R C}$ mice $(n=7-9)$. Data are mean \pm SEM. A ${ }^{*} P<0.05,{ }^{*} P<0.01$ by 2-tailed Student's $t$ test.

of renal inflammation, injury, and fibrosis were also improved in $\mathrm{mAb}$-PA-treated mice (Figure 6C). These observations confirmed that pharmacologic blockage of PAPP-A enzymatic activity ameliorates disease progression in ADPKD mice.

\section{Discussion}

Previously, we have reported that nutritional manipulations ameliorate ADPKD (18). The beneficial effects of FR and FR-like diets appear to be mediated by suppression of the IGF pathway. The IGF pathway is one of the main growth factor pathways, playing a key role in several physiological and pathological conditions $(23,24)$. Surprisingly, little is known about the role of the IGF pathway in the pathogenesis of ADPKD. In fact, while it has previously been speculated that IGF-1 may play a role in ADPKD $(29,30-32$, 84), no causal or mechanistic in vivo data directly implicate any of the components of the IGF pathway in cystic disease. In the present study, we determined for the first time to our knowledge a direct role for IGF and its regulatory components in ADPKD, and demonstrated that PAPP-A plays a role in the pathogenesis of ADPKD.

Higher expression of PAPP-A was observed in humans with versus without $A D P K D$, and in experimental ADPKD mice compared with controls. Interestingly, FR, which reduced cystic disease in the murine model, also significantly reduced Pappa mRNA expression. PAPP-A has been shown to correlate with renal function, be present in higher levels in patients on dialysis, and serve as an independent predictor of mortality of patients on hemodialysis $(49,50,51)$. PAPP-A is also a marker of inflammation in dialysis patients $(85,86)$. Mice deficient in PAPP-A also show resistance against diabetic nephropathy (87). Interestingly, our ADPKD mouse model defi-

cient in PAPP-A showed a significant reduction in kidney size and cystic burden, with a remarkable reduction in renal inflammation, injury, and fibrosis compared with ADPKD mice. Kidney function and survival were improved even with fewer copies of PAPP-A in PKD mice. Although ADPKD-PAPP-A KO mice were smaller in size compared with littermates, the effects of the PAPP-A gene occurred independently of its effect on animal and tissue size. Since we used animal body weight and heart weight for normalization, the effect of decreased size and weight could not account for all the effects of PAPP-A deficiency on PKD, including kidney inflammation, injury, and cystic index. Furthermore, we focused GFR measurements in ADPKD-PAPP-A WT versus ADPKD-PAPP-A-heterozygous mice to overcome any potential confounding effect of the smaller size of ADPKD-PAPP-A KO mice. Surprisingly, we found that even one fewer 
copy of PAPP-A in PKD mice significantly reduced GFR, leading to improved kidney function. These findings clearly demonstrated a role for PAPP-A in the pathogenesis of experimental ADPKD. The reduction in cystic burden appeared to be mediated by a reduction in proliferation, not apoptosis or autophagy.

Although the role of the cAMP pathway in the pathogenesis of ADPKD is supported by several lines of evidence $(4,6,7,59-62)$, it is not completely known how mechanisms downstream of the cAMP pathway lead to the development of cystic disease in vivo. cAMP levels are increased in ADPKD and have been shown to regulate both cellular proliferation and fluid secretion, but there are no reports to our knowledge of the regulation of PAPP-A expression by cAMP in any cell type except non-kidney cells $(63,64)$. Interestingly, we observed that while PAPP-A expression was induced by FSK in experimental ADPKD — including cultured human ADPKD cells and murine models of ADPKD and metanephros - it was not greatly induced by FSK in normal human kidney cells or WT mice. In vivo, it cannot be excluded that the cAMP/ PKA pathway cooperates with other pathways to raise PAPP-A. Although this could be mediated by a higher increase in cAMP levels in ADPKD cells, it appears unlikely, since IGFBP4 expression is similarly stimulated by cAMP in control and ADPKD cells, and relative PKA activity is similar in normal and PKD cells. ERK and calcium signaling do not seem to be involved in regulation of PAPP-A expression. Although AMPK activation and hypermethylation treatment were able to abolish cAMP-induced PAPP-A expression in PKD cells, inhibition of AMPK and hypomethylation failed to increase cAMP-induced PAPP-A expression in normal cells to the same extent as observed in PKD cells. Thus, it appears that the expression of PAPP-A in PKD could be regulated through several signaling pathways and/or epigenetically through a specific modification in the PAPP-A gene that makes it more responsive to cAMP stimulation in ADPKD cells and tissues. miRNAs also have been implicated in the regulation of PAPP-A expression (88) and could be involved in its regulation in PKD too. Further studies need to be performed to confirm this.

Previous studies have shown that the proliferation of cyst epithelial cells in ADPKD is induced by the cAMP pathway in a PKA-dependent manner (89). In autosomal recessive polycystic kidney disease (ARPKD), both PKA and Epac have been shown to activate cholangiocyte proliferation and hepatic cystogenesis by the MEK/ERK1/2 pathway (90). However, in the present study we found that the regulation of PAPP-A by cAMP is only partially mediated by Epac in ADPKD. Our studies identified that the main pathway downstream of cAMP that regulates PAPP-A expression in ADPKD is mediated by PKA/CREB/p300. The metanephric culture was used to demonstrate the importance of the IGF-1/IGFBP4/PAPP-A pathway in cystogenesis in the presence of FSK but without insulin supplementation, in contrast to the protocol used in previous studies (59). In our studies, we did not use insulin in the culture, and therefore in the absence of any growth factors (insulin or IGF), cyst formation was not observed in FSK-only-treated metanephroi, but cystogenesis occurred in the presence of both IGF-1 and cAMP, demonstrating that combination with a growth factor (IGF or insulin) is needed for the effect of FSK on cystic formation in WT metanephric kidneys. When $P k d 1^{R C / R C}$ mice were used for metanephric culture, cystogenesis was observed even when IGF-1 was lacking in the media, indicating that availability of endogenous growth factor may drive cystic formation induced by PKA in PKD mice.

Although the cAMP pathway is complex, and is likely to crosstalk with several other signaling pathways, our studies have delineated the role of distinct downstream components of the cAMP pathway in the regulation of both PAPP-A expression and cystogenesis in ADPKD. In conclusion, our study demonstrates a direct role of the IGF/IGFBP/PAPP-A pathway in ADPKD. Our study opens potential new avenues for the development of treatment options for this devastating disease.

\section{Methods}

Supplemental Methods are available online with this article.

Animal studies. Mice of the $P k d 1^{R C / R C}$ (PKD1 p.R3277C) model of ADPKD (C57BL/6J background) were generated in-house (82). PAPP-A-deficient ADPKD mice were generated by crossing PAPP-A-deficient Pappa ${ }^{t m I C a c}$ mice (81) with the $P k d 1^{R C / R C}$ mice. C57BL/6J mice were purchased from the Jackson Laboratory. Both male and female mice were used in the study. Animals were housed in standard cages (5 mice per cage) in an animal housing room maintained at constant temperature and humidity with 12-hour light/12 hour dark cycles. For PAPP-A antibody experiments, $P k d 1^{R C / R C}$ mice were given weekly i.p. injections of 30 mg/kg mAb-PA (provided by Ansh Labs) (91) or control IgG (R\&D Systems) for 6 weeks. For FSK studies, a single dose of FSK ( $5 \mathrm{mg} / \mathrm{kg}$ ) or vehicle control (5\% DMSO) was administered by i.p. injection. For CBP inhibitor studies, $P k d 1^{R C / R C}$ mice were treated with GNE- $049(30 \mathrm{mg} / \mathrm{kg})$ or vehicle $(5 \% \mathrm{DMSO}, 5 \%$ ethanol, $30 \%$ PEG 400, and 60\% Phosol 50 PG) twice a day for 3 days. 
Blood was collected for biochemical analysis, and organs were weighed. Portions of tissue were placed in formalin and processed for histological studies or snap frozen in liquid nitrogen and stored at $-80^{\circ} \mathrm{C}$ for protein or gene expression analysis.

Reagents and antibodies. All reagents and chemicals were purchased from Sigma-Aldrich, unless otherwise specified. Recombinant human IGF-I and IGFBP4 were from R\&D Systems. Antibodies to p-ERK (catalog 4370), ERK(catalog 4695), p-Akt (catalog 4060), Akt (catalog 4691), P-IGFR1 (catalog 3918), IGFR1 (catalog 3027), p-AMPK (catalog 2532), AMPK (catalog 2535) were purchased from Cell Signaling Technology. PCNA antibody (sc-7907) was from Santa Cruz Biotechnology. Polyclonal PAPP-A antibodies for Western blotting (92) and immunostaining (PAC1-D8-mIgG2a) (93) were used. The PAPP-A(pico) ELISA kit was a gift from Ansh Labs.

BUN measurement. BUN was measured using an Abaxis Vetscan VS2 machine (Abaxis Inc.) as described previously (94).

Cystatin $C$ measurement. Blood was collected from the posterior vena cava into heparinized tubes at the time of sacrifice and plasma was separated out by centrifugation. Plasma cystatin $C$ levels were assessed using a Mouse/Rat Cystatin C Quantikine ELISA Kit (R\&D Systems) according to the manufacturer's instructions.

GFR measurement. GFR was measured in conscious mice by the FITC-inulin method as described previously (95). Briefly, $5 \%$ FITC-inulin prepared in $0.85 \% \mathrm{NaCl}$ solution was dialyzed for 24 hours. The concentration of dialyzed FITC-inulin was calculated, and then the solution was filtered through $0.22-\mu \mathrm{m}$ filter. The filtered FITC-inulin solution was injected in mice retro-orbitally at $2 \mu \mathrm{L} / \mathrm{g}$ body weight. Blood was collected from mouse tail at 3, 5, 7, 10, 15, 35, 56, and 75 minutes after injection in Na-heparin minicapillaries. Plasma was diluted 1:10 in 0.5 mol/L HEPES (pH 7.4), and fluorescence was measured with a NanoDrop 3300 (Thermo Fisher Scientific).

Culture of metanephroi. Metanephros organ culture was performed as described previously $(59,83)$, except that insulin was not added to the media. Metanephroi were isolated from C57BL6/J mice on embryonic day 13.5 and placed on transparent Falcon $0.4-\mu \mathrm{m}$ cell culture inserts in 12 -well plates (Fisher Scientific) at $37^{\circ} \mathrm{C}$ in a humidifier incubator $\left(5 \% \mathrm{CO}_{2}\right)$ in serum-free medium. Metanephroi were grown in DMEM/F12 medium supplemented with $5 \mu \mathrm{g} / \mathrm{mL}$ transferrin, $2.8 \mathrm{nM}$ selenium, $25 \mathrm{ng} / \mathrm{mL}$ prostaglandin $\mathrm{E}_{1}$, and $6 \mathrm{ng} / \mathrm{ml} \mathrm{T} 3,1 \%$ penicillin/streptomycin, and medium was changed every 2 days. Cysts were generated by treatment with FSK $(10 \mu \mathrm{M})$, and development of cysts was observed every day. For antibody neutralization experiment, IGF-1 (6.5 nM), IGFBP4 (26 nM), and PAPP-A (320 pM) were added to the culture after 4 hours treatment with FSK.

Histological and immunohistochemical analysis. Formalin-fixed, paraffin-embedded tissues were cut into $5-\mu \mathrm{m}$-thick cross sections and stained with H\&E by the Mayo Histology Core Laboratory for cystic index analysis. Cystic index (cyst area proportional to cross-sectional kidney area) was measured as described previously (18). Immunostaining for CD3 was performed using anti-CD3 antibody (1:100, Agilent Technologies, A0452). Fibrosis was assessed by Sirius red staining. The percentages of areas positive for fibrosis and anti-CD3 were calculated using ImageJ (NIH).

Human ADPKD kidney tissue slides were produced in-house under an institutionally approved IRB protocol, and normal human kidney tissue slides were purchased from Novus Biologicals.

Western blot analysis. Western blot analysis on kidney tissues and cultured cells was performed as described earlier (18). Membranes were stripped and probed with actin, tubulin, or GAPDH antibody to control for equal gel loading and transfer. Films were scanned, and densitometry was performed using ImageJ.

$R T-P C R$. Total RNA was isolated using the RNeasy Plus Mini Kit (QIAGEN), and cDNA was prepared using the QuantiTect Reverse Transcription Kit (QIAGEN) or High-Capacity cDNA Reverse Transcription Kit (Applied Biosystems). Commercially available TaqMan gene expression probes were obtained from Applied Biosystems, and RT-PCR was performed in duplicate as described previously (18). The relative mRNA expression of target genes was calculated using the $2^{-\mathrm{ddCq}}$ method with Gapdh as internal reference gene.

Cell culture. RCTE and 9-12 cells were cultured in DMEM:F12 with 10\% FBS and 1\% penicillin/streptomycin. Cells were treated with $10 \mu \mathrm{M}$ FSK, $200 \mu \mathrm{M}$ 6-MB-cAMP, or $30 \mu \mathrm{M}$ 8CPT2OMe for 16 hours and RNA was isolated. Cells were also treated with $10 \mu \mathrm{M}$ FSK in the presence or absence of $100 \mu \mathrm{M}$ Rp cAMPS for 16 hours or different dosage of KG-501 and RNA was isolated. RCTE and 9-12 cells were also treated with $10 \mu \mathrm{M} \mathrm{CBP30}$ for 24 hours followed by RNA isolation. 9-12 Cells were pretreated with $200 \mu \mathrm{M}$ A769661 or DMSO for 6 hours, followed by FSK treatment for 16 hours and RNA was isolated. PAPP-A protein levels in cell-free conditioned medium and also in human cystic fluid (collected at the Mayo Clinic with approval by the IRB) was measured with the PAPP-A(pico) ELISA kit. 
PAPP-A-mediated IGFBP4 proteolysis assay. The PAPP-A-mediated IGFBP4 proteolysis assay in RCTE and 9-12 cells was performed as described previously (96). Briefly, human IGFBP4 precomplexed with IGF was incubated in cell-free conditioned medium with or without inhibitory PAPP-A monoclonal antibody (mAb-PA 1/41) for 72 hours at $37^{\circ} \mathrm{C}$. For in vivo determination of PAPP-A activity, human IGFBP4 precomplexed with or without IGF was incubated with kidney membrane fractions for 72 hours at $37^{\circ} \mathrm{C}$. Western blot and quantitative analysis was done as described previously (96).

Statistics. Data are expressed as mean \pm SEM. Comparisons were made by unpaired Student's $t$ test and ANOVA. Nonparametric tests were used wherever required by data distribution. $P$ values less than 0.05 were considered significant. Survival analysis was done using Kaplan-Meier curves.

Study approval. Human cystic fluid was collected and human ADPKD kidney tissue slides were produced under Mayo Clinic IRB-approved protocols that included written informed consent from all patients. All animal experimental protocols were approved by the Institutional Animal Care and Use Committee of Mayo Clinic (A47715 and A00003864), and studies were conducted in accordance with the NIH Guide for the Care and Use of Laboratory Animals (National Academies Press, 2011). Both male and female mice were used for the studies.

\section{Author contributions}

SK and KZH conducted and analyzed the in vivo work. SK, KZH, CCSC, and GMW performed and analyzed the in vitro work. LKB and JL performed immunohistochemistry studies in the human kidney. LKB performed proteolytic assays and PAPP-A ELISAs. KZH, SK, and ENC wrote the manuscript. ENC designed experiments, analyzed results, and supervised the project. VET, $\mathrm{PCH}, \mathrm{CO}$, and CAC provided the resources and revised the manuscript. SK and KZH contributed equally to this work based on the dedication to the design, performance and analysis of experiments.

\section{Acknowledgments}

Jessica Gronniger's help with mouse genotyping is acknowledged. We thank Ansh Labs for the gift of the PAPP-A(pico) ELISA kit. This work was supported in part by grants from the Mayo Clinic Center for Biomedical Discovery and grant DK090728 from the Mayo Translational PKD Center.

Address correspondence to author: Eduardo N. Chini, 222 3rd Ave. SW, Rochester, Minnesota, USA 55905. Phone: 507.284.6696; Email: chini.eduardo@mayo.edu.

Kyaw Zaw Hein's present address is: Rush University Medical Center, Chicago, Illinois, USA.

1. Tan YC, Blumenfeld J, Rennert H. Autosomal dominant polycystic kidney disease: genetics, mutations and microRNAs. Biochim Biophys Acta. 2011;1812(10):1202-1212.

2. Torres VE, Harris PC, Pirson Y. Autosomal dominant polycystic kidney disease. Lancet. 2007;369(9569):1287-1301.

3. Chebib FT, Torres VE. Autosomal dominant polycystic kidney disease: core curriculum 2016. Am J Kidney Dis. 2016;67(5):792-810.

4. Bolignano D, Palmer SC, Ruospo M, Zoccali C, Craig JC, Strippoli GFM. Interventions for preventing the progression of autosomal dominant polycystic kidney disease. Cochrane Database Syst Rev. 2015;(7):CD010294.

5. Grantham JJ, Geiser JL, Evan AP. Cyst formation and growth in autosomal dominant polycystic kidney disease. Kidney Int. 1987;31(5):1145-1152.

6. Harris PC. 2008 Homer W. Smith Award: insights into the pathogenesis of polycystic kidney disease from gene discovery. $J A m$ Soc Nephrol. 2009;20(6):1188-1198.

7. Rowe I, Boletta A. Defective metabolism in polycystic kidney disease: potential for therapy and open questions. Nephrol Dial Transplant. 2014;29(8):1480-1486.

8. Mair W, Dillin A. Aging and survival: the genetics of life span extension by dietary restriction. Annu Rev Biochem. 2008;77:727-754.

9. Fontana L, Partridge L, Longo VD. Extending healthy life span — from yeast to humans. Science. 2010;328(5976):321-326.

10. Podrini C, et al. Dissection of metabolic reprogramming in polycystic kidney disease reveals coordinated rewiring of bioenergetic pathways. Commun Biol. 2018;1:194.

11. Chiaravalli M, et al. 2-Deoxy-d-glucose ameliorates PKD progression. J Am Soc Nephrol. 2016;27(7):1958-1969.

12. Rowe I, et al. Defective glucose metabolism in polycystic kidney disease identifies a new therapeutic strategy. Nat Med. 2013;19(4):488-493

13. Riwanto M, Kapoor S, Rodriguez D, Edenhofer I, Segerer S, Wüthrich RP. Inhibition of Aerobic glycolysis attenuates disease progression in polycystic kidney disease. PLOS ONE. 2016;11(1):e0146654

14. Menezes LF, Lin CC, Zhou F, Germino GG. Fatty acid oxidation is impaired in an orthologous mouse model of autosomal dominant polycystic kidney disease. EBioMedicine. 2016;5:183-192.

15. Lin CC, et al. A cleavage product of polycystin-1 is a mitochondrial matrix protein that affects mitochondria morphology and function when heterologously expressed. Sci Rep. 2018;8(1):2743. 
16. Padovano V, et al. The polycystins are modulated by cellular oxygen-sensing pathways and regulate mitochondrial function. $M o l$ Biol Cell. 2017;28(2):261-269.

17. Padovano V, Podrini C, Boletta A, Caplan MJ. Metabolism and mitochondria in polycystic kidney disease research and therapy Nat Rev Nephrol. 2018;14(11):678-687.

18. Warner G, et al. Food restriction ameliorates the development of polycystic kidney disease. J Am Soc Nephrol. 2016;27(5):1437-1447.

19. Kipp KR, Rezaei M, Lin L, Dewey EC, Weimbs T. A mild reduction of food intake slows disease progression in an orthologous mouse model of polycystic kidney disease. Am J Physiol Renal Physiol. 2016;310(8):F726-F731.

20. Torres JA, et al. Ketosis ameliorates renal cyst growth in polycystic kidney disease. Cell Metab. 2019;30(6):1007-1023.e5.

21. Brandhorst S, et al. A periodic diet that mimics fasting promotes multi-system regeneration, enhanced cognitive performance, and healthspan. Cell Metab. 2015;22(1):86-99.

22. VanHook AM. Periodic dieting promotes health. Science Signaling. 2015;8(385):ec187.

23. Anisimov VN. Insulin/IGF-1 signaling pathway driving aging and cancer as a target for pharmacological intervention. Exp Gerontol. 2003;38(10):1041-1049.

24. LeRoith D, Roberts CT Jr. The insulin-like growth factor system and cancer. Cancer Lett. 2003;195(2):127-137.

25. Brahmkhatri VP, Prasanna C, Atreya HS. Insulin-like growth factor system in cancer: novel targeted therapies. Biomed Res Int. 2015;2015:538019.

26. Christopoulos PF, Msaouel P, Koutsilieris M. The role of the insulin-like growth factor-1 system in breast cancer. Mol Cancer $2015 ; 14: 43$

27. Bach LA, Hale LJ. Insulin-like growth factors and kidney disease. Am J Kidney Dis. 2015;65(2):327-336.

28. Parker E, et al. Insulin-like growth factor-1 induces hyperproliferation of PKD1 cystic cells via a Ras/Raf dependent signalling pathway. Kidney Int. 2007;72(2):157-165.

29. Song X, et al. Systems biology of autosomal dominant polycystic kidney disease (ADPKD): computational identification of gene expression pathways and integrated regulatory networks. Hum Mol Genet. 2009;18(13):2328-2343.

30. Aukema HM, Housini I. Dietary soy protein effects on disease and IGF-I in male and female Han:SPRD-cy rats. Kidney Int. 2001;59(1):52-61.

31. Nakamura T, et al. Growth factor gene expression in kidney of murine polycystic kidney disease. J Am Soc Nephrol. 1993;3(7):1378-1386.

32. Liu C, Zhang Y, Yuan L, Fu L, Mei C. Rosiglitazone inhibits insulin-like growth factor-1-induced polycystic kidney disease cell growth and p70S6 kinase activation. Mol Med Rep. 2013;8(3):861-864.

33. Zhou R, Diehl D, Hoeflich A, Lahm H, Wolf E. IGF-binding protein-4: biochemical characteristics and functional consequences. J Endocrinol. 2003;178(2):177-193.

34. Oxvig C. The role of PAPP-A in the IGF system: location, location, location. J Cell Commun Signal. 2015;9(2):177-187.

35. Conover CA. Key questions and answers about pregnancy-associated plasma protein-A. Trends Endocrinol Metab. 2012;23(5):242-249.

36. Swindell WR, Masternak MM, Bartke A. In vivo analysis of gene expression in long-lived mice lacking the pregnancy-associated plasma protein A (PappA) gene. Exp Gerontol. 2010;45(5):366-374.

37. Adamo ML, Ma X, Ackert-Bicknell CL, Donahue LR, Beamer WG, Rosen CJ. Genetic increase in serum insulin-like growth factor-I (IGF-I) in C3H/HeJ compared with C57BL/6J mice is associated with increased transcription from the IGF-I exon 2 promoter. Endocrinology. 2006;147(6):2944-2955.

38. Resch ZT, Simari RD, Conover CA. Targeted disruption of the pregnancy-associated plasma protein-A gene is associated with diminished smooth muscle cell response to insulin-like growth factor-I and resistance to neointimal hyperplasia after vascular injury. Endocrinology. 2006;147(12):5634-5640.

39. Miller BS, et al. Pregnancy associated plasma protein-A is necessary for expeditious fracture healing in mice. J Endocrinol. 192(3):505-513.

40. Hourvitz A, et al. The regulated expression of the pregnancy-associated plasma protein-A in the rodent ovary: a proposed role in the development of dominant follicles and of corpora lutea. Endocrinology. 2002;143(5):1833-1844.

41. Conover CA, et al. Pregnancy-associated plasma protein-a is the insulin-like growth factor binding protein- 4 protease secreted by human ovarian granulosa cells and is a marker of dominant follicle selection and the corpus luteum. Endocrinology. 2001;142(5):2155.

42. Rehage M, et al. Transgenic overexpression of pregnancy-associated plasma protein-A increases the somatic growth and skeletal muscle mass in mice. Endocrinology. 2007;148(12):6176-6185.

43. Bulut I, et al. Relationship between pregnancy-associated plasma protein-A and lung cancer. Am J Med Sci. 2009;337(4):241-244.

44. Conover CA, Oxvig C. PAPP-A and cancer. J. Mol Endocrinol. 2018;61(1):T1-T10.

45. Conover CA, Bale LK, Mader JR, Mason MA, Keenan KP, Marler RJ. Longevity and age-related pathology of mice deficient in pregnancy-associated plasma protein-A. J Gerontol A Biol Sci Med Sci. 2010;65(6):590-599.

46. Li X, Liu Q, Zhou T, Zhao S, Zhou S. PAPP-A: a possible pathogenic link to the instability of atherosclerotic plaque. Med Hypotheses. 2008;70(3):597-599.

47. Harrington SC, Simari RD, Conover CA. Genetic deletion of pregnancy-associated plasma protein-A is associated with resistance to atherosclerotic lesion development in apolipoprotein E-deficient mice challenged with a high-fat diet. Circ Res. 2007;100(12):1696-1702.

48. Conover CA, et al. Transgenic overexpression of pregnancy-associated plasma protein-A in murine arterial smooth muscle accelerates atherosclerotic lesion development. Am J Physiol Heart Circ Physiol. 2010;299(2):H284-H291.

49. Etter C, et al. Pregnancy-associated plasma protein-A is an independent short-time predictor of mortality in patients on maintenance haemodialysis. Eur Heart J. 2010;31(3):354-359.

50. Kalousová M, Benáková H, Kuběna AA, Dusilová-Sulková S, Tesař V, Zima T. Pregnancy-associated plasma protein A as an independent mortality predictor in long-term hemodialysis patients. Kidney Blood Press Res. 2012;35(3):192-201.

51. Kalousová M, Tesaír V, Muravská A, Zima T. Pregnancy-associated plasma protein A: spotlight on kidney diseases. Clin Chem Lab Med. 2012;50(7):1183-1190. 
52. Lawrence JB, et al. The insulin-like growth factor (IGF)-dependent IGF binding protein-4 protease secreted by human fibroblasts is pregnancy-associated plasma protein-A. Proc Natl Acad Sci U S A. 1999;96(6):3149-3153.

53. Qin X, Byun D, Strong DD, Baylink DJ, Mohan S. Studies on the role of human insulin-like growth factor-II (IGF-II)-dependent IGF binding protein (hIGFBP)-4 protease in human osteoblasts using protease-resistant IGFBP-4 analogs. J Bone Miner Res. 1999;14(12):2079-2088.

54. Bayes-Genis A, et al. Insulin-like growth factor binding protein-4 protease produced by smooth muscle cells increases in the coronary artery after angioplasty. Arterioscler Thromb Vasc Biol. 2001;21(3):335-341.

55. Bayes-Genis A, et al. Pregnancy-associated plasma protein A as a marker of acute coronary syndromes. $N$ Engl J Med. 2001;345(14):1022-1029.

56. Conover CA, Oxvig C, Overgaard MT, Christiansen M, Giudice LC. Evidence that the insulin-like growth factor binding protein-4 protease in human ovarian follicular fluid is pregnancy associated plasma protein-A. J Clin Endocrinol Metab. 1999;84(12):4742-4745.

57. Bonno M, et al. Localization of pregnancy-associated plasma protein-A and colocalization of pregnancy-associated plasma protein-A messenger ribonucleic acid and eosinophil granule major basic protein messenger ribonucleic acid in placenta. Lab Invest. 1994;71(4):560-566.

58. Loghman-Adham M, Nauli SM, Soto CE, Kariuki B, Zhou J. Immortalized epithelial cells from human autosomal dominant polycystic kidney cysts. Am J Physiol Renal Physiol. 2003;285(3):F397-F412.

59. Magenheimer BS, et al. Early embryonic renal tubules of wild-type and polycystic kidney disease kidneys respond to camp stimulation with cystic fibrosis transmembrane conductance regulator/ $\mathrm{Na}+, \mathrm{K}+, 2 \mathrm{Cl}-$ co-transporter-dependent cystic dilation. J Am Soc Nephrol. 2006;17(12):3424-3437.

60. Torres VE, Wang X, Qian Q, Somlo S, Harris PC, Gattone VH. Effective treatment of an orthologous model of autosomal dominant polycystic kidney disease. Nat Med. 2004;10(4):363-364.

61. Sutters M, et al. Polycystin-1 transforms the cAMP growth-responsive phenotype of M-1 cells. Kidney Int. 2001;60(2):484-494.

62. Torres VE, Harris PC. Strategies targeting cAMP signaling in the treatment of polycystic kidney disease. J Am Soc Nephrol. 2014;25(1):18-32.

63. Haaning J, Oxvig C, Overgaard MT, Ebbesen P, Kristensen T, Sottrup-Jensen L. Complete cDNA sequence of the preproform of human pregnancy-associated plasma protein-A. Evidence for expression in the brain and induction by cAMP. Eur J Biochem. 1996;237(1):159-163.

64. Conover CA, Chen BK, Resch ZT. Regulation of pregnancy-associated plasma protein-A expression in cultured human osteoblasts. Bone. 2004;34(2):297-302.

65. Mikkelsen JH, et al. Indirect targeting of IGF receptor signaling in vivo by substrate-selective inhibition of PAPP-A proteolytic activity. Oncotarget. 2014;5(4):1014-1025

66. Yan K, Gao LN, Cui YL, Zhang Y, Zhou X. The cyclic AMP signaling pathway: exploring targets for successful drug discovery (Review). Mol Med Rep. 2016;13(5):3715-3723.

67. Cheng X, Ji Z, Tsalkova T, Mei F. Epac and PKA: a tale of two intracellular cAMP receptors. Acta Biochim Biophys Sin (Shanghai). 2008;40(7):651-662.

68. Vuchak LA, Tsygankova OM, Prendergast GV, Meinkoth JL. Protein kinase A and B-Raf mediate extracellular signal-regulated kinase activation by thyrotropin. Mol Pharmacol. 2009;76(5):1123-1129.

69. Misra UK, Pizzo SV. Upregulation of mTORC2 activation by the selective agonist of EPAC, 8-CPT-2Me-cAMP, in prostate cancer cells: assembly of a multiprotein signaling complex. J Cell Biochem. 2012;113(5):1488-500.

70. Gjertsen B, et al. Novel (Rp)-cAMPS analogs as tools for inhibition of cAMP-kinase in cell culture: basal cAMP-kinase activity modulates interleukin-1 $\beta$ action. J Biol Chem. 1995;270(35):20599-20607.

71. Ye H, et al. The regulatory $1 \alpha$ subunit of protein kinase A modulates renal cystogenesis. Am J Physiol Renal Physiol. 2017;313(3):F677-FF86.

72. Williams SS, et al. Tissue-specific regulation of the mouse Pkhd1 (ARPKD) gene promoter. Am J Physiol Renal Physiol. 2014;307(3):F356-F368.

73. Best JL, et al. Identification of small-molecule antagonists that inhibit an activator: coactivator interaction. Proc Natl Acad Sci U $S$ A. 2004;101(51):17622-17627.

74. Vo N, Goodman RH. CREB-binding protein and p300 in transcriptional regulation. J Biol Chem. 2001;276(17):13505-13508

75. Hammitzsch A, et al. CBP30, a selective CBP/p300 bromodomain inhibitor, suppresses human Th17 responses. Proc Natl Acad Sci U S A. 2015;112(34):10768-10773.

76. Jin L, et al. Therapeutic targeting of the $\mathrm{CBP} / \mathrm{p} 300$ bromodomain blocks the growth of castration-resistant prostate cancer. Cancer Res. 2017;77(20):5564-5575.

77. Ning J, Clemmons DR. AMP-activated protein kinase inhibits IGF-I signaling and protein synthesis in vascular smooth muscle cells via stimulation of insulin receptor substrate $1 \mathrm{~S} 794$ and tuberous sclerosis 2 S1345 phosphorylation. Mol Endocrinol. 2010;24(6):1218-1229.

78. Göransson O, et al. Mechanism of action of A-769662, a valuable tool for activation of AMP-activated protein kinase. $J$ Biol Chem. 2007;282(45):32549-32560.

79. Morris EJ, et al. Discovery of a novel ERK inhibitor with activity in models of acquired resistance to BRAF and MEK inhibitors. Cancer Discov. 2013;3(7):742-750.

80. Loddo M, et al. Pregnancy-associated plasma protein A regulates mitosis and is epigenetically silenced in breast cancer. J Pathol. 2014;233(4):344-356.

81. Conover CA, et al. Metalloproteinase pregnancy-associated plasma protein A is a critical growth regulatory factor during fetal development. Development. 2004;131(5):1187-1194.

82. Hopp K, et al. Functional polycystin-1 dosage governs autosomal dominant polycystic kidney disease severity. J Clin Invest. 2012;122(11):4257-4273.

83. Barak H, Boyle SC. Organ culture and immunostaining of mouse embryonic kidneys. Cold Spring Harb Protoc. 2011;2011(1):pdb. prot5558. 
84. Parker E, et al. Hyperproliferation of PKD1 cystic cells is induced by insulin-like growth factor-1 activation of the Ras/Raf signalling system. Kidney Int. 2007;72(2):157-165.

85. Coskun A, et al. Pregnancy-associated plasma protein A in dialysis patients. Clin Chem Lab Med. 2007;45(1):63-66.

86. Kalousová M, et al. Increased levels of pregnancy-associated plasma protein A are associated with mortality in hemodialysis patients: preliminary results. Blood Purif. 2004;22(3):298-300.

87. Mader JR, et al. Mice deficient in PAPP-A show resistance to the development of diabetic nephropathy. J Endocrinol. 2013;219(1):51-58.

88. Sun Y, et al. MiR-490-3p modulates the proliferation of vascular smooth muscle cells induced by ox-LDL through targeting PAPP-A. Cardiovasc Res. 2013;100(2):272-279.

89. Yamaguchi T, et al. cAMP stimulates the in vitro proliferation of renal cyst epithelial cells by activating the extracellular signal-regulated kinase pathway. Kidney Int. 2000;57(4):1460-1471

90. Banales JM, Masyuk TV, Gradilone SA, Masyuk AI, Medina JF, LaRusso NF. The cAMP effectors Epac and protein kinase a (PKA) are involved in the hepatic cystogenesis of an animal model of autosomal recessive polycystic kidney disease (ARPKD). Hepatology. 2009;49(1):160-174.

91. Becker MA, Haluska P, Bale LK, Oxvig C, Conover CA. A novel neutralizing antibody targeting pregnancy-associated plasma protein-a inhibits ovarian cancer growth and ascites accumulation in patient mouse tumorgrafts. Mol Cancer Ther. 2015;14(4):973-981.

92. Oxvig C, Sand O, Kristensen T, Kristensen L, Sottrup-Jensen L. Isolation and characterization of circulating complex between human pregnancy-associated plasma protein-A and proform of eosinophil major basic protein. Biochim Biophys Acta. 1994;1201(3):415-423.

93. Mikkelsen JH, Steffensen LB, Oxvig C. Development of a recombinant antibody towards PAPP-A for immunohistochemical use in multiple animal species. J Immunol Methods. 2014;404:33-40.

94. Kilari S, Yang B, Sharma A, McCall DL, Misra S. Increased transforming growth factor beta (TGF- $\beta$ ) and pSMAD3 signaling in a murine model for contrast induced kidney injury. Sci Rep. 2018;8(1):6630

95. Rieg T. A high-throughput method for measurement of glomerular filtration rate in conscious mice. JVis Exp. 2013(75):e50330.

96. Bale LK, West SA, Conover CA. Characterization of mouse pericardial fat: regulation by PAPP-A. Growth Horm IGF Res. 2018;42-43:1-7. 